\title{
Flashover of a vacuum-insulator interface: A statistical model
}

\author{
W. A. Stygar, ${ }^{1}$ H. C. Ives, ${ }^{2}$ T. C. Wagoner, ${ }^{3}$ J. A. Lott, ${ }^{3}$ V. Anaya,${ }^{3}$ H. C. Harjes, ${ }^{1}$ J. P. Corley, ${ }^{3}$ R. W. Shoup,,${ }^{1}$ D. L. Fehl, ${ }^{1}$ \\ G. R. Mowrer, ${ }^{1}$ Z. R. Wallace, ${ }^{3}$ R. A. Anderson, ${ }^{1}$ J. D. Boyes, ${ }^{1}$ J.W. Douglas, ${ }^{4}$ M. L. Horry, ${ }^{1}$ T. F. Jaramillo, ${ }^{3}$ \\ D. L. Johnson, ${ }^{4}$ F.W. Long, ${ }^{1}$ T. H. Martin, ${ }^{1}$ D. H. McDaniel, ${ }^{1}$ O. Milton, ${ }^{1}$ M. A. Mostrom, ${ }^{5}$ D. A. Muirhead, ${ }^{1}$ \\ T. D. Mulville, ${ }^{3}$ J. J. Ramirez, ${ }^{1}$ L. E. Ramirez, ${ }^{5}$ T. M. Romero, ${ }^{3}$ J. F. Seamen, ${ }^{1}$ J.W. Smith, ${ }^{1}$ C. S. Speas, ${ }^{1}$ R. B. Spielman, ${ }^{1}$ \\ K.W. Struve, ${ }^{1}$ G. E. Vogtlin, ${ }^{6}$ D. E. Walsh, ${ }^{7}$ E. D. Walsh, ${ }^{7}$ M. D. Walsh, ${ }^{7}$ and O. Yamamoto ${ }^{8}$ \\ ${ }^{1}$ Sandia National Laboratories, Albuquerque, New Mexico 87185, USA \\ ${ }^{2} E G \& G$, Albuquerque, New Mexico 87107, USA \\ ${ }^{3}$ Ktech Corporation, Albuquerque, New Mexico 87123, USA \\ ${ }^{4}$ Titan-Pulsed Sciences Division, San Leandro, California 94577, USA \\ ${ }^{5}$ Misson Research Corporation, Albuquerque, New Mexico 87110, USA \\ ${ }^{6}$ Lawrence Livermore National Laboratory, Livermore, California 94550, USA \\ ${ }^{7}$ C-Lec Plastics, Philadelphia, Pennsylvania 19135, USA \\ ${ }^{8}$ Kyoto University, Kyoto, Japan
}

(Received 5 March 2004; published 23 July 2004)

\begin{abstract}
We have developed a statistical model for the flashover of a $45^{\circ}$ vacuum-insulator interface (such as would be found in an accelerator) subject to a pulsed electric field. The model assumes that the initiation of a flashover plasma is a stochastic process, that the characteristic statistical component of the flashover delay time is much greater than the plasma formative time, and that the average rate at which flashovers occur is a power-law function of the instantaneous value of the electric field. Under these conditions, we find that the flashover probability is given by $1-\exp \left(-E_{p}^{\beta} t_{\mathrm{eff}} C / k^{\beta}\right)$, where $E_{p}$ is the peak value in time of the spatially averaged electric field $E(t), t_{\mathrm{eff}} \equiv \int\left[E(t) / E_{p}\right]^{\beta} d t$ is the effective pulse width, $C$ is the insulator circumference, $k \propto \exp (\lambda / d)$, and $\beta$ and $\lambda$ are constants. We define $E(t)$ as $V(t) / d$, where $V(t)$ is the voltage across the insulator and $d$ is the insulator thickness. Since the model assumes that flashovers occur at random azimuthal locations along the insulator, it does not apply to systems that have a significant defect, i.e., a location contaminated with debris or compromised by an imperfection at which flashovers repeatedly take place, and which prevents a random spatial distribution. The model is consistent with flashover measurements to within $7 \%$ for pulse widths between $0.5 \mathrm{~ns}$ and $10 \mu \mathrm{s}$, and to within a factor of 2 between $0.5 \mathrm{~ns}$ and $90 \mathrm{~s}$ (a span of over 11 orders of magnitude). For these measurements, $E_{p}$ ranges from 64 to $651 \mathrm{kV} / \mathrm{cm}, d$ from 0.50 to $4.32 \mathrm{~cm}$, and $C$ from 4.96 to $95.74 \mathrm{~cm}$. The model is significantly more accurate, and is valid over a wider range of parameters, than the J. C. Martin flashover relation that has been in use since 1971 [J. C. Martin on Pulsed Power, edited by T. H. Martin, A. H. Guenther, and M. Kristiansen (Plenum, New York, 1996)]. We have generalized the statistical model to estimate the total-flashover probability of an insulator stack (i.e., an assembly of insulator-electrode systems connected in series). The expression obtained is consistent with the measured flashover performance of a stack of five 5.72-cm-thick, 1003-cm-circumference insulators operated at 100 and $158 \mathrm{kV} / \mathrm{cm}$. The expression predicts that the total-flashover probability is a strong function of the ratio $E_{p} / k$, and that under certain conditions, the performance improves as the capacitance between the stack grading rings is increased. In addition, the expression suggests that given a fixed stack height, there exists an optimum number of insulator rings that maximizes the voltage at which the stack can be operated. The results presented can be applied to any system (or any set of systems connected in series) subject to random failures, when the characteristic statistical delay time of a failure is much greater than its formative time.
\end{abstract}

DOI: 10.1103/PhysRevSTAB.7.070401

PACS numbers: $84.70 .+\mathrm{p}, 77.22 . J p, 52.80 .-\mathrm{s}, 52.90 .+\mathrm{z}$

\section{INTRODUCTION}

The operation of pulsed-power and particle accelerators often requires that a high-voltage pulse be transmitted across a vacuum-insulator interface. Because of various technical considerations, it is usually desired to minimize the size of the interface, and to estimate $E_{p}$, the peak value in time of the spatially averaged electric-field $E(t)$, at which such an interface can be operated reliably for the duration of the pulse. [We define $E(t)$ as $V(t) / d$, where $V(t)$ is the voltage across the insulator and $d$ is the insulator thickness.] More specifically, it is desired to estimate the insulator-flashover probability as a function of $E_{p}$, the temporal width of the electric-field pulse $E(t)$, and the dimensions of the interface.

Since 1964, the standard vacuum-insulator interface for pulsed applications has consisted of a $45^{\circ}$ insulatorelectrode system, or several such systems connected in series [1-39]. A single $45^{\circ}$ system is outlined in Fig. 1. (By convention, the interface depicted in this figure is 


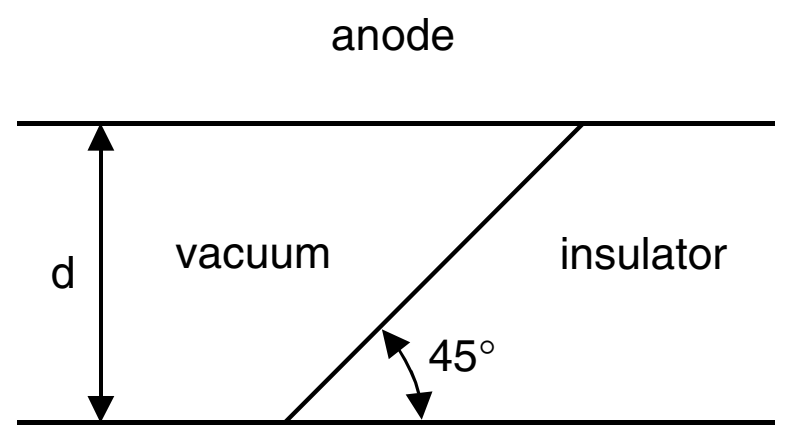

\section{cathode}

FIG. 1. Idealized $45^{\circ}$ vacuum-insulator interface. (In a $-45^{\circ}$ configuration, the bottom electrode in the above configuration would be the anode.)

defined to be in a $45^{\circ}$ configuration. In a $-45^{\circ}$ configuration, the lower electrode in Fig. 1 would be the anode.) A typical assembly of several $45^{\circ}$ insulator-electrode systems connected in series, i.e., an insulator stack [1], is shown in Fig. 2. This stack is one of four that form the vacuum interface of the 20-MA, 50-TW $Z$ pulsed-power accelerator [31,32,34-36,40-44].

An expression for the peak electric field $E_{p}$ at which a single $45^{\circ}$ insulator is expected to flash was first developed by Martin [6,29]. The J. C. Martin (JCM) expression estimates the flashover field as a function of an effective electric-field pulse width and the vacuum-insulatorinterface area. The expression assumes that the insulator material is polymethyl methacrylate (PMMA), also referred to as acrylic. Even though the JCM relation was intended only as a first approximation [6,29], it has been used as the standard in the international pulsed-power community since 1971. It has also been generalized to estimate the flashover probability of a $45^{\circ}$ vacuum-insulator interface [30,35].

However, the JCM expression does not appear to have a strong experimental foundation. As discussed in Appendix A, of the eight flashover measurements used to develop the JCM relation [6,29], only six are independent, and of these, only two are directly relevant to the design of a pulsed-power accelerator such as $Z$. In addition, as discussed in Appendix A, the pulse-width scaling predicted by JCM appears to be inconsistent with more recent experiments.

To improve the accuracy of estimated flashover probabilities, we develop in Sec. IIA an insulator-flashover relation based on a statistical-model of the flashover process. The model was first developed by Zutavern, Buttram, and O'Malley for millisecond-pulse water breakdown [45], and was subsequently applied to vacuum-insulator-flashover in Ref. [36].

In Sec. IIB, we normalize the statistical model to 17 PMMA flashover measurements obtained over a wide range of experimental parameters. Most of these

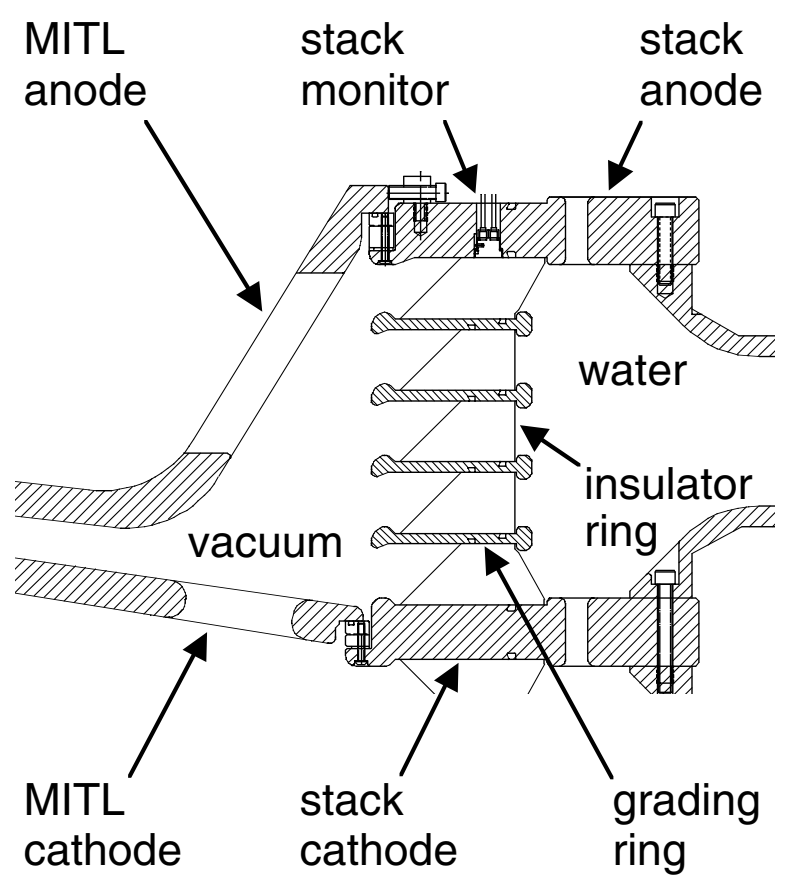

FIG. 2. One of the four insulator stacks of the 20-MA, 50TW $Z$ pulsed-power accelerator. The stack consists of five $45^{\circ}$ 5.72-cm-thick, 1003-cm-circumference Rexolite insulator rings connected in series, and forms part of the accelerator's vacuum interface. The figure shows the anode and cathode of the magnetically insulated transmission line (MITL) that is normally connected to the stack. The hardware is cylindrically symmetric about an axis to the left of the figure. Six $D$-dot and three $B$-dot monitors are located in the stack anode at nine azimuthally equidistant locations; an outline of one of the monitors is shown here.

experiments were performed after the JCM flashover relation was developed.

In Sec. IIC, we compare the JCM expression to the statistical model, and to the results of the flashover measurements.

The statistical model (developed in Secs. IIA and IIB) assumes that the time delay between the application of a voltage across an insulator, and the completion of a flashover, is dominated by the statistical component of the delay time. The statistical component is the time between the application of the voltage and the appearance of the free electron or electrons that initiate the formation of the flashover plasma. The model assumes that the time between the initiation and completion of the plasma formation-the formative component - can be neglected. The work presented in Secs. IIA and IIB complements flashover models that describe the plasma formation, after the flashover has been initiated. Such models are described in $[3,9,13,15,46-66]$, references therein, and the review article by Anderson [24]. Two of these models are outlined in Sec. IID.

Sections IIA and IIB use the statistical model to calculate the flashover probability for a single insulator ring. 
In Sec. IIIA1, we generalize this result to develop a formal expression for the total-flashover probability of an insulator stack.

The calculations developed in Sec. IIIA1 extend the work presented in Refs. [30,36]. The calculations in Sec. IIIA1 differ from those described by Smith [30], since Smith assumes JCM scaling. In addition, Ref. [30] uses an expression for the flashover probability that is valid only when the probability is small, and an expression for the differential probability that is valid only when the electric-field time history is a square pulse. We do not make these approximations; hence the calculations in Sec. IIIA1 are valid for arbitrary values of the probability and arbitrary time histories. Furthermore, Ref. [30] defines the effective width of the electricfield pulse to be the full width at $89 \%$ of its peak value $[6,29]$. The flashover model in Sec. IIIA1 leads to a different definition of pulse width that is self-consistent and valid for arbitrary pulse shapes. Reference [30] also estimates the probability that two insulators in a multiinsulator stack flash, and assumes that this is approximately equivalent to a total-stack flashover. Hence the calculations in [30] cannot estimate the full benefit of using a stack with a large number of insulators. In Sec. IIIA1, we do not make this assumption, and instead calculate the probability that all of the insulators in a stack flash.

The formal expression for the total-stack-flashover probability given in Sec. IIIA1 can be integrated directly, but this approach becomes increasingly tedious as the number of insulators in a stack increases. In Sec. IIIA2 we develop an alternate and considerably simpler analytic expression.

Sections IIIA1 and IIIA2 make the simplifying assumption that each time an insulator ring in a stack flashes, the resulting increased electric field across the remaining unflashed rings appears instantaneously around the entire circumference of the stack, i.e., that the azimuthal transit time of a flashover voltage pulse can be neglected. Following ideas described by Smith [30], we estimate in Sec. IIIA3 and Appendix B effects due to a nonzero transit time.

Sections IIIA1 and IIIA2 also assume that before any of the rings in a stack flash, the voltage across the stack is divided evenly among the insulator rings, and that each time a ring flashes, the voltage is divided evenly among the remaining unflashed rings. Following ideas presented by Smith [30], we estimate the effects of uneven voltage division in Sec. IIIA4 and Appendix C.

In Sec. IIIA5, we suggest a method for taking into account both the effects of nonzero transit time and uneven voltage division.

In Sec. IIIB, we present analytic expressions for the probability that none of the insulators in a stack flash, and that at least one insulator flashes. These auxiliary flashover probabilities are considerably simpler than those presented in Sec. IIIA, and can in certain situations provide meaningful information.

In Sec. IVA, we compare the predictions of Secs. II and III with the observed performance of one of the insulator stacks of the $Z$ pulsed-power accelerator, and demonstrate that within experimental uncertainties, the predictions of the statistical model are consistent with the observations. In Sec. IVB, we discuss two methods that can be used to improve the flashover performance of a stack.

We discuss limits of applicability of the statistical model in Sec. VA. In Sec. VB, we note that the relations developed in Secs. II and III can be applied to any system (or any set of systems connected in series) subject to random failures, when the characteristic statistical delay time of a failure is much greater than its formative time.

\section{FLASHOVER OF A SINGLE INSULATOR RING}

\section{A. Statistical model}

\section{The single-ring-flashover probability $f(t)$}

In this section, we develop a theoretical flashover relation for a single insulator ring. The relation assumes that the flashover process can be described by the statistical model first developed by Zutavern, Buttram, and O'Malley for millisecond-pulse water breakdown [45], and subsequently applied to the flashover of insulators in vacuum [36]. A similar statistical model has been used to describe the pulsed electrical breakdown of spark gaps [67-72].

We begin with the simplifying assumption that the characteristic time delay between the application of a voltage to a vacuum-insulator interface, and the completion of a flashover, can be expressed as follows [45,67-72]:

$$
\tau_{\text {delay }}=\tau_{\text {stat }}+\tau_{\text {form}} .
$$

We define the statistical component of the delay time $\tau_{\text {stat }}$ to be the characteristic time between the application of the voltage and the appearance of the free electron or electrons that initiate the formation of the flashover plasma. We define $\tau_{\text {form }}$ to be the formative component: the time between the initiation and completion of the plasma formation. (Seminal measurements of the formative time for the pulsed electrical breakdown of a spark gap are described by Fletcher [73].)

We consider situations for which

$$
\tau_{\text {stat }} \gg \tau_{\text {form }}
$$

i.e., when $\tau_{\text {delay }}$ is dominated by $\tau_{\text {stat }}$, and $\tau_{\text {form }}$ can be neglected [45]. Equation (2) is consistent with $45^{\circ}$-insulator-flashover measurements presented by Anderson in Fig. 7 of Ref. [11]. This figure shows that under the conditions studied in [11], the flashover delay can vary from 1.5 to $100 \mathrm{~ns}$. This suggests that $\tau_{\text {delay }}$ is dominated by $\tau_{\text {stat }}$, since $\tau_{\text {form }}$ is apparently $\leq 1.5 \mathrm{~ns}$. As discussed in 
Sec. IIB, we estimate that for these experiments, $\tau_{\text {delay }} \geq$ $7.7 \tau_{\text {form }}$. Equation (2) is also consistent with results presented by Yamamoto and co-workers [20,22,23], which indicate that for the $45^{\circ}$ system investigated, flashovers occur between 2 and $10 \mu$ s after the beginning of the applied voltage pulse. (The delay times are shorter for the experiments described in [11] than in [20,22,23], because the electric fields are higher in [11].) For these results $[20,22,23]$, we estimate (as discussed in Sec. IIB) that $\tau_{\text {delay }} \geq 14 \tau_{\text {form }}$. Hence it appears that for the configurations studied by Anderson [11] and Yamamoto and coworkers [20,22,23], Eq. (2) is a reasonable approximation. In addition, as we demonstrate in Secs. IIB and IVA, Eq. (2) leads to a flashover relation that is consistent with other $45^{\circ}$-insulator-flashover measurements conducted over a wide range of experimental parameters.

To develop the statistical relation, we consider a large population of identical $45^{\circ}$ circular insulator rings, each of which is subject to an applied unipolar voltage pulse $V(t)$, where $V(t)=0$ for $t \leq 0$. We define

$$
E(t) \equiv \frac{V(t)}{d}
$$

to be the spatially averaged electric field across each insulator, where $d$ is the insulator thickness as indicated in Fig. 1. We assume that the polarity of the pulse is such that the upper electrode in Fig. 1 is the anode. We assume also that the thickness $d$ is the same for each of the insulator rings in the population, and that the vacuuminsulator interface of each ring has unit circumferential length. (Throughout this article, we define the circumference of an insulator to be that at the midplane of its vacuum-insulator interface.)

We define $N_{\text {unit }}(0)$ to be the size of the population, and $N_{\text {unit }}(t)$ to be the number of insulators that survive until time $t$ without flashing. When Eq. (2) is valid, the average insulator-flashover rate at any time during the voltage pulse is independent of past history. Hence (for a given value of $d$ ) the flashover rate can be a function only of the instantaneous value of $E(t)$. We assume that the flashover rate is a monotonically increasing function of $E(t)$, and that this function can be approximated as a power law:

$$
\frac{1}{N_{\text {unit }}(t)} \frac{\partial N_{\text {unit }}(t)}{\partial t}=-\left(\frac{E(t)}{k}\right)^{\beta}
$$

Since we assume that the flashover rate is independent of past history, $\beta$ and $k$ must be independent of time. We assume also that $k$ is a (as yet unspecified) function of $d$. Integrating Eq. (4) gives

$$
s_{\text {unit }}(t) \equiv \frac{N_{\text {unit }}(t)}{N_{\text {unit }}(0)}=\exp \left[-\frac{1}{k^{\beta}} \int_{0}^{t} E^{\beta}(\tau) d \tau\right],
$$

where $s_{\text {unit }}(t)$ is the probability of survival until time $t$.

To extend Eq. (5) to insulators with arbitrary circumferential length, we consider a large population of insu- lator rings, each with circumference $C$ and thickness $d$, subject to the electric field $E(t)$. We define $N(0)$ to be the size of the population, and $N(t)$ to be the number of insulators that survive until time $t$ without flashing. We model each insulator as $C$ unit-length insulators in parallel. Assuming that the survival of a unit-length segment does not affect the survival of any other segment, the survival probability of an insulator with circumference $C$ can be expressed as

$$
s(t) \equiv \frac{N(t)}{N(0)}=s_{\text {unit }}^{C}(t)
$$

Since Eq. (6) assumes that flashovers occur at random azimuthal locations along the insulator, it does not apply to systems that have a significant defect, i.e., a location contaminated with debris or compromised by an imperfection at which flashovers repeatedly take place, and which prevents a random spatial distribution.

Combining Eqs. (5) and (6) gives the flashover (failure) probability $f(t)$ :

$$
\begin{aligned}
f(t) & =1-s(t)=1-\exp \left[\frac{-C}{k^{\beta}} \int_{0}^{t} E^{\beta}(\tau) d \tau\right] \\
& =1-\exp \left(-\frac{E_{p}^{\beta} t_{\mathrm{eff}} C}{k^{\beta}}\right),
\end{aligned}
$$

where $E_{p}$ is the peak value in time of the electric field $E(t)$, and the effective pulse width $t_{\text {eff }}$ is defined as follows:

$$
t_{\mathrm{eff}} \equiv \frac{1}{E_{p}^{\beta}} \int_{0}^{t} E^{\beta}(\tau) d \tau
$$

Equation (7) can be considered as an exponential distribution with variate $E_{p}^{\beta} t_{\mathrm{eff}} C / k^{\beta}$, or as a Weibull distribution [74] with variate $E_{p}\left(t_{\mathrm{eff}} C\right)^{1 / \beta} / k$ and shape parameter $\beta$.

Equation (7) is based on Eqs. (1)-(6), and predicts that the flashover probability $f(t)$ is a function of the product $t_{\text {eff }} C$. When flashovers occur randomly in both time and space, as is assumed by Eq. (7), the variables $t_{\text {eff }}$ and $C$ play equal roles [45]. For example, when $f(t) \ll 1$, Eq. (7) predicts that $f(t) \propto t_{\text {eff }} C$.

We consider briefly a large population of identical dielectric-electrode systems for which Eq. (2) is not valid, and for which instead $\tau_{\text {stat }} \ll \tau_{\text {form }}$. Assuming that statistical fluctuations in $\tau_{\text {form }}$ can be neglected, electrode-surface conditions are the same for each system, and the dielectrics are homogeneous and defect-free, then all of the dielectrics in a population of such systems would fail at approximately the same time. The failure probability $f(t)$ would to a good approximation equal 0 when $t<\tau_{\text {form }}$, and 1 when $t>\tau_{\text {form }}$. In general, $\tau_{\text {form }}$ would be a function of the electric-field topology, applied-voltage time history, and dielectric and electrode materials. Under these ideal conditions, $f(t)$ would be 
independent of the circumference or area of the electrodes.

\section{The 50\% and average flashover fields}

According to Eq. (7), the flashover probability $f(t)$ equals $50 \%$ when

$$
\frac{E_{p}^{\beta}\left(t_{\mathrm{eff}} C\right)}{k^{\beta}}=\ln 2
$$

As discussed in the previous section, we assume $k$ is a function of the insulator thickness $d$ (Fig. 1). To determine this functional dependence, we consider the flashover measurements performed by Yamamoto and co-workers $[20,22,23]$. These results, which are summarized in Table I, give the peak electric field $E_{p}$ at which the flashover probability equals $50 \%$ for insulators with $d=$ $0.5,1.0$, and $1.5 \mathrm{~cm}$. We determine empirically from these measurements that the $50 \%$ flashover field is approximately proportional to $\exp (\lambda / d)$, where $\lambda$ is a constant, and express the condition for a $50 \%$ flashover probability
[Eq. (9)] as follows:

$$
\frac{E_{p}\left(t_{\mathrm{eff}} C\right)^{1 / \beta}}{\exp (\lambda / d)}=\gamma_{\mathrm{SM}}
$$

where $\gamma_{\mathrm{SM}}$ is a constant, and

$$
k \equiv \frac{\gamma_{\mathrm{SM}} \exp (\lambda / d)}{(\ln 2)^{1 / \beta}} .
$$

Consequently, to estimate the flashover probability for a single ring $f(t)$ [Eq. (7)], we must know $\beta, \lambda$, and the statistical-model constant $\gamma_{\mathrm{SM}}$.

As discussed in Sec. IIB below, we estimate these constants by normalizing Eq. (10) to a subset of the flashover measurements presented in Table I. Each of the measurements listed in this table was obtained with an uncoated $45^{\circ}$ PMMA or cross-linked polystyrene (Rexolite) insulator between flat uncoated electrodes, at a pressure $\leq 10^{-4}$ torr $[7,8,11,17-23,39]$. The measurements were made with no external sources of charged particles or ultraviolet radiation, and a magnetic field due

TABLE I. Summary of insulator-flashover measurements. Each of these was obtained with an uncoated $45^{\circ}$ polymethylmethacrylate (PMMA) or cross-linked polystyrene (Rexolite) insulator between flat uncoated electrodes, at a pressure $\leq$ $10^{-4}$ torr. The measurements were made with no external sources of charged particles or ultraviolet radiation, and a magnetic field due only to the displacement current that charged the capacitance defined by the insulator and electrodes. The sixth and tenth columns list for each measurement the JCM and statistical-model constants, $\gamma_{\mathrm{JCM}}$ and $\gamma_{\mathrm{SM}}$, respectively. The constants assume $E_{p}$

\begin{tabular}{|c|c|c|c|c|c|c|c|c|c|}
\hline Reference & Insulator material & $E_{p}(\mathrm{kV} / \mathrm{cm})$ & $t_{89 \%}(\mu \mathrm{s})$ & $A\left(\mathrm{~cm}^{2}\right)$ & $\gamma_{\mathrm{JCM}}$ & $t_{\text {eff }}(\mu \mathrm{s})$ & $C(\mathrm{~cm})$ & $d(\mathrm{~cm})$ & $\gamma_{\mathrm{SM}}$ \\
\hline Anderson [11] & PMMA & 405 & 0.0100 & 20 & 254 & 0.00979 & 23.6 & 0.6 & 234 \\
\hline Jaitly and Sudarshan $[17,18]$ & PMMA & 70.7 & $9 \times 10^{7}$ & 6.46 & 1804 & $9 \times 10^{7}$ & 4.96 & 0.92 & 399 \\
\hline Jaitly and Sudarshan $[17,18]$ & PMMA & 73.9 & $9 \times 10^{7}$ & 9.48 & 1959 & $9 \times 10^{7}$ & 7.29 & 0.92 & 434 \\
\hline Jaitly and Sudarshan $[17,18]$ & PMMA & 64.1 & $9 \times 10^{7}$ & 11.16 & 1727 & $9 \times 10^{7}$ & 8.58 & 0.92 & 382 \\
\hline Jaitly and Sudarshan $[17,18]$ & PMMA & 81.5 & $9 \times 10^{7}$ & 13.98 & 2246 & $9 \times 10^{7}$ & 10.74 & 0.92 & 497 \\
\hline Milton [8] & PMMA & 1100 & 0.000778 & 5.36 & 395 & 0.000631 & 14.91 & 0.254 & 268 \\
\hline Milton [8] & PMMA & 651 & 0.000495 & 10.14 & 231 & 0.000507 & 14.11 & 0.508 & 248 \\
\hline Milton [8] & PMMA & 523 & 0.000920 & 14.35 & 213 & 0.000739 & 13.31 & 0.762 & 240 \\
\hline Milton [8] & PMMA & 444 & 0.00152 & 17.98 & 201 & 0.00112 & 12.52 & 1.016 & 229 \\
\hline Milton [8] & PMMA & 417 & 0.00178 & 21.05 & 197 & 0.00147 & 11.72 & 1.27 & 230 \\
\hline Milton [8] & Rexolite & 417 & 0.00178 & 21.05 & 197 & 0.00147 & 11.72 & 1.27 & 230 \\
\hline Milton [8] & PMMA & 346 & 0.00880 & 24.09 & 216 & 0.00736 & 10.71 & 1.59 & 231 \\
\hline Milton [7] & PMMA & 313 & 0.00797 & 21.05 & 190 & 0.00627 & 11.72 & 1.27 & 200 \\
\hline Milton [7] & PMMA & 365 & 0.164 & 5.36 & 319 & 0.134 & 14.91 & 0.254 & 152 \\
\hline Milton [7] & PMMA & 315 & 0.181 & 10.14 & 299 & 0.163 & 14.11 & 0.508 & 213 \\
\hline Milton [7] & PMMA & 278 & 0.433 & 14.35 & 316 & 0.330 & 13.31 & 0.762 & 235 \\
\hline Milton [7] & PMMA & 242 & 0.619 & 17.98 & 298 & 0.487 & 12.52 & 1.016 & 229 \\
\hline Milton [7] & PMMA & 196 & 0.658 & 21.05 & 248 & 0.519 & 11.72 & 1.27 & 194 \\
\hline Milton [7] & Rexolite & 223 & 0.902 & 21.05 & 297 & 0.723 & 11.72 & 1.27 & 229 \\
\hline Stygar et al. [39] & PMMA & 265 & 0.00927 & 584.6 & 230 & 0.00734 & 95.74 & 4.318 & 242 \\
\hline Stygar et al. [39] & Rexolite & 293 & 0.0175 & 584.6 & 282 & 0.0141 & 95.74 & 4.318 & 286 \\
\hline Vogtlin et al. $[19,21]$ & PMMA & 522 & 0.00103 & 7.33 & 202 & 0.000836 & 10.37 & 0.5 & 201 \\
\hline Vogtlin et al. $[19,21]$ & PMMA & 471 & 0.00103 & 10.16 & 189 & 0.000836 & 9.58 & 0.75 & 211 \\
\hline Yamamoto et al. $[20,22,23]$ & PMMA & 238 & 11.8 & 5.55 & 426 & 10.0 & 7.85 & 0.5 & 228 \\
\hline Yamamoto et al. $[20,22,23]$ & PMMA & 179 & 11.8 & 13.33 & 350 & 10.0 & 9.43 & 1.0 & 222 \\
\hline Yamamoto et al. $[20,22,23]$ & PMMA & 164 & 11.8 & 23.33 & 339 & 10.0 & 11.00 & 1.5 & 224 \\
\hline
\end{tabular}
is expressed in $\mathrm{kV} / \mathrm{cm}, t_{89 \%}$ and $t_{\text {eff }}$ in $\mu \mathrm{s}, A$ in $\mathrm{cm}^{2}$, and $C, d$ in $\mathrm{cm}$. The quantity $A$ is the total area of the vacuum-insulator interface, the circumference $C$ is that at the midplane of the vacuum-insulator interface, and the thickness $d$ is as defined by Fig. 1 . 
only to the displacement current that charged the capacitance defined by the insulator and electrodes. The parameters listed in the table were inferred from the information provided in Refs. [7,8,11,17-23,39,75,76].

As discussed above, the flashover fields listed in Table I that were obtained by Yamamoto and co-workers $[20,22,23]$ are those at which the flashover probability $f(t)$ is approximately $50 \%$. According to Eq. (10), the field at which $f(t)$ equals $50 \%$ is given by

$$
E_{p}(50 \%)=\frac{\gamma_{\mathrm{SM}} \exp (\lambda / d)}{\left(t_{\mathrm{eff}} C\right)^{1 / \beta}} .
$$

The other flashover fields listed in Table I are not 50\% values, but average values obtained from several measurements. Assuming $t_{\text {eff }}$ is approximately constant for each set of measurements performed with a given insulator configuration, we find that for the flashoverprobability distribution given by Eq. (7), the average flashover field can be expressed as [77]

$$
E_{p}(\operatorname{avg})=\frac{\Gamma\left(1+\frac{1}{\beta}\right)}{(\ln 2)^{1 / \beta}} \frac{\gamma_{\mathrm{SM}} \exp (\lambda / d)}{\left(t_{\mathrm{eff}} C\right)^{1 / \beta}}=\frac{\Gamma\left(1+\frac{1}{\beta}\right)}{(\ln 2)^{1 / \beta}} E_{p}(50 \%),
$$

where $\Gamma$ is the gamma function [78]. As discussed in Sec. IIB below, $\beta$ is approximately equal to 10 . Hence as indicated by Eq. (13), the difference between the $50 \%$ and average flashover fields is $1.3 \%$ [77,78]. Since this difference is negligible compared to experimental errors, we treat these fields as being equivalent.

\section{B. The constants $\beta, \lambda$, and $\gamma_{\mathrm{SM}}$}

To determine the constants $\beta, \lambda$, and $\gamma_{\mathrm{SM}}$ we performed a numerical least-squares analysis. We set each flashover field in Table I equal to the $50 \%$ value, and used Eq. (10) to calculate for each flashover measurement $\gamma_{\mathrm{SM}}$ as a function of $\beta$ and $\lambda$, for $8 \leq \beta \leq 12$ and $0.15 \leq \lambda \leq$ $0.33 \mathrm{~cm}$.

For the 17 PMMA measurements listed in Table I for which $0.5 \mathrm{~ns} \leq t_{\mathrm{eff}} \leq 10 \mu \mathrm{s}$ and $0.5 \leq d \leq 4.32 \mathrm{~cm}$, we find that the standard deviation in the values of $\gamma_{\mathrm{SM}}$ is minimized when

$$
\begin{gathered}
\beta=10 \pm 1.2, \\
\lambda=0.24 \pm 0.08 \mathrm{~cm} .
\end{gathered}
$$

The uncertainties given in Eqs. (14) and (15) are estimated as follows. Since we use 17 measurements, we find that the $1 \sigma$ fractional uncertainty in the standard deviation of the values of $\gamma_{\mathrm{SM}}$ is $[2(17-1)]^{-1 / 2}=18 \%$ [79]. When $\lambda$ is held fixed at $0.24 \mathrm{~cm}, \mathrm{a}+18 \%,-0 \%$ fractional variation in the standard deviation of $\gamma_{\mathrm{SM}}$ is obtained when $\beta$ is varied by \pm 1 .2. When $\beta$ is held fixed at 10 , a $+18 \%,-0 \%$ fractional variation in the standard deviation is obtained when $\lambda$ is varied by $\pm 0.08 \mathrm{~cm}$. (We note

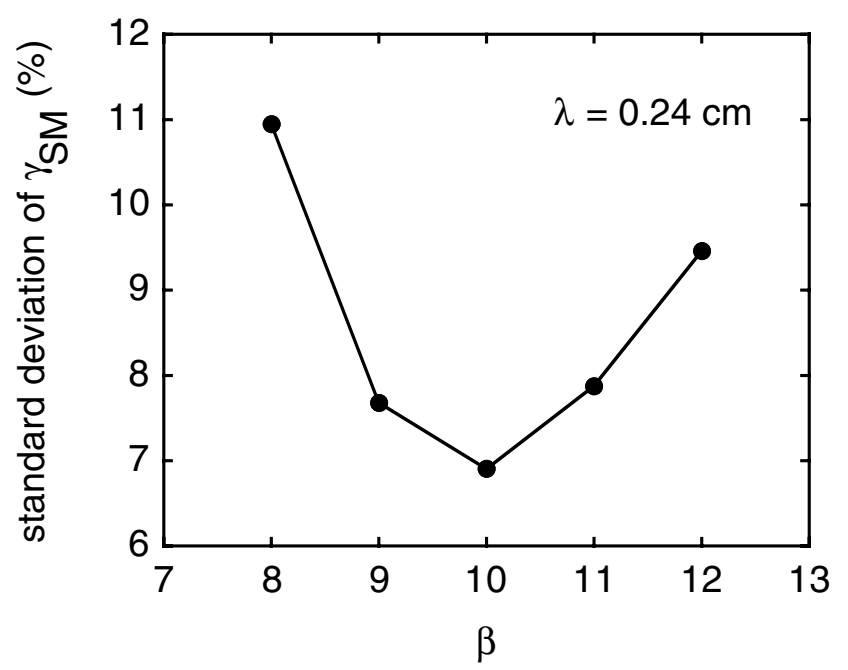

FIG. 3. The fractional standard deviation of the values of $\gamma_{\mathrm{SM}}$ as a function of $\beta$, for the 17 PMMA measurements listed in Table I with $0.5 \mathrm{~ns} \leq t_{\mathrm{eff}} \leq 10 \mu \mathrm{s}$ and $0.5 \leq d \leq 4.32 \mathrm{~cm}$. The plot assumes $\lambda=0.24 \mathrm{~cm}$.

that in general, variations in $\beta$ and $\lambda$ are statistically correlated [79].)

A subset of the results of the least-squares analysis is presented in Figs. 3 and 4. Figure 3 plots the fractional standard deviation in the values of $\gamma_{\mathrm{SM}}$ as a function of $\beta$ when $\lambda=0.24 \mathrm{~cm}$; Fig. 4 plots the standard deviation as a function of $\lambda$ when $\beta=10$. When $\beta=10$ and $\lambda=$ $0.24 \mathrm{~cm}$, the average value of $\gamma_{\mathrm{SM}}$ is 224 and the standard deviation is $15(7 \%)$. Hence we estimate that the flashover probability is approximately $50 \%$ when

$$
\frac{E_{p}\left(t_{\mathrm{eff}} C\right)^{1 / 10}}{\exp (0.24 / d)}=\gamma_{\mathrm{SM}}=224 \pm 15(\mathrm{PMMA})
$$

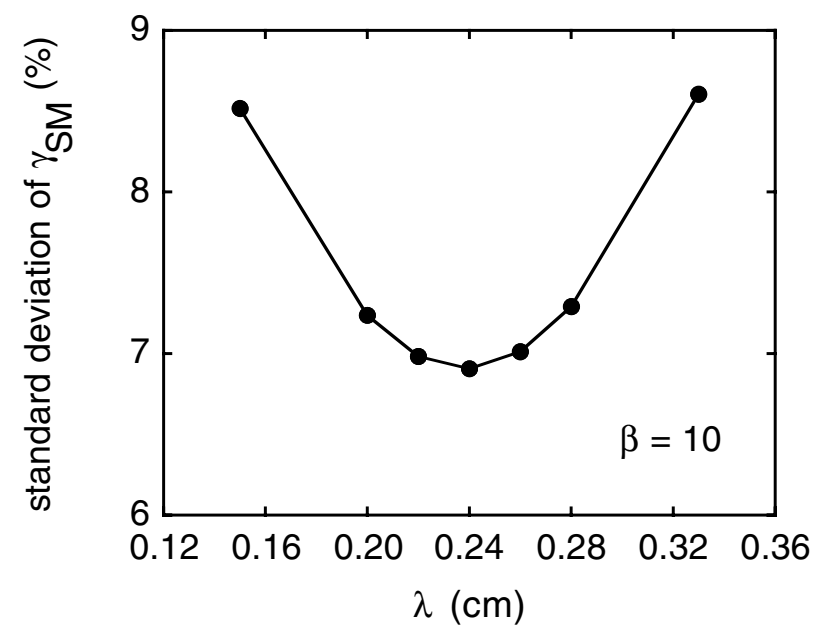

FIG. 4. The fractional standard deviation of the values of $\gamma_{\mathrm{SM}}$ as a function of $\lambda$, for the 17 PMMA measurements listed in Table I with $0.5 \mathrm{~ns} \leq t_{\text {eff }} \leq 10 \mu$ s and $0.5 \leq d \leq 4.32 \mathrm{~cm}$. The plot assumes $\beta=10$. 
Following convention, Eq. (16) assumes $E_{p}$ is expressed in $\mathrm{kV} / \mathrm{cm}, t_{\text {eff }}$ in $\mu \mathrm{s}$, and $C$ and $d$ in $\mathrm{cm}$. As indicated earlier, we define the circumference $C$ to be that at the midplane of the vacuum-insulator interface, and $d$ as shown in Fig. 1.

As discussed in Sec. IIA1, Eq. (16) assumes $\tau_{\text {delay }} \sim$ $\tau_{\text {stat }} \gg \tau_{\text {form }}$ [Eq. (2)]. For the experiments described by Anderson [11], we find $\tau_{\text {delay }} \geq 7.7 \tau_{\text {form }}$. To obtain this relation, we estimate that $\tau_{\text {delay }} \sim t_{\text {eff }}$, where $t_{\text {eff }}$ is given in Table I. To estimate $\tau_{\text {form }}$, we assume that the statistical delay is negligible on the shots taken by Anderson for which the total-flashover delay is at the minimum observed value, use Eq. (8) to estimate for these shots the effective pulse width, and assume that this width is an upper bound on $\tau_{\text {form }}$. For the conditions investigated by Yamamoto and co-workers $[20,22,23]$, we find in a similar manner that $\tau_{\text {delay }} \geq 14 \tau_{\text {form. }}$. We note that Eq. (16) provides a reasonable approximation for $E_{p}$ when $\tau_{\text {form }}$ is as large as $0.5 \tau_{\text {stat }}$, since in this case the error in $\tau_{\text {delay }} \sim$ $t_{\text {eff }}$ is $50 \%$, and in $E_{p}, 4 \%$.

In Fig. 5, we plot $E_{p}$ as a function of $\left(t_{\mathrm{eff}} C\right)^{-1 / 10} \exp (0.24 / d)$ for conditions under which the flashover probability is approximately $50 \%$. We plot the 17 PMMA measurements with $0.5 \mathrm{~ns} \leq t_{\text {eff }} \leq 10 \mu$ s and $0.5 \leq d \leq 4.32 \mathrm{~cm}$, and the four measurements listed in Table I with $t_{\text {eff }}=90 \mathrm{~s}$. Even though Eq. (16) was developed using the PMMA data for which $0.5 \mathrm{~ns} \leq t_{\mathrm{eff}} \leq$ $10 \mu \mathrm{s}$ and $0.5 \leq d \leq 4.32 \mathrm{~cm}$, it predicts to within a factor of 2 the flashover fields for the 90-s measurements. In other words, Eq. (16) is consistent (to within a factor of 2) with experiments for pulse widths that span over 11 orders of magnitude.

Table I lists only three measurements performed with Rexolite. Assuming that the scaling indicated by Eq. (16) applies to Rexolite as well, we estimate that the flashover probability equals 50\% for a single Rexolite insulator ring when

$$
\frac{E_{p}\left(t_{\mathrm{eff}} C\right)^{1 / 10}}{\exp (0.24 / d)}=\gamma_{\mathrm{SM}} \sim 248 \text { (Rexolite). }
$$

Equations (16) and (17) suggest that the flashover strength of Rexolite is $\sim 11 \%$ higher than that of PMMA. We note that relative flashover measurements performed by Roth and co-workers $[33,80]$ also suggest that the flashover strength of Rexolite is $11 \%$ higher than that of PMMA, although given the uncertainties in Eqs. (16) and (17), such agreement must be considered fortuitous. The first measurements to suggest that Rexolite is comparable or superior to PMMA were performed by Milton [7,8]. (In Fig. 5 of Ref. [7], Rexolite is referred to as C Lecstyrene.)

\section{Comparison of the JCM flashover relation to the statistical model}

The JCM insulator-flashover relation $[6,29]$ is described in Appendix A. To permit a comparison of the

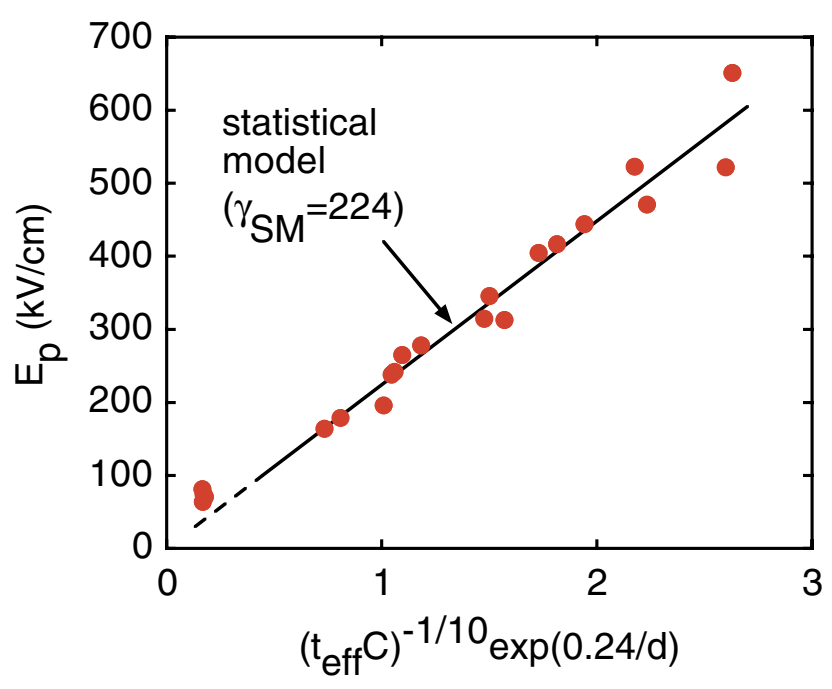

FIG. 5. (Color) The peak electric field $E_{p}$ as a function of $\left(t_{\text {eff }} C\right)^{-1 / 10} \exp (0.24 / d)$, which is the statistical-model scaling, for conditions under which the flashover probability is approximately $50 \%$. (The horizontal axis assumes $t_{\text {eff }}$ is expressed in $\mu \mathrm{s}$, and $C, d$ in $\mathrm{cm}$.) The figure plots the 17 PMMA measurements listed in Table I with $0.5 \mathrm{~ns} \leq t_{\mathrm{eff}} \leq 10 \mu \mathrm{s}$ and $0.5 \leq$ $d \leq 4.32 \mathrm{~cm}$, and the four measurements with $t_{\text {eff }}=90 \mathrm{~s}$. This is the same set of measurements used for Fig. 6. The fractional standard deviation in the values of $\gamma_{\mathrm{SM}}$ for the 17 measurements is $7 \%$. The 90 -s measurements lie close together, and are the only measurements with $E_{p}<100 \mathrm{kV} / \mathrm{cm}$. The model becomes inapplicable as either $t_{\text {eff }} \rightarrow \infty$ or $C \rightarrow \infty$, which are equivalent to the limit $E_{p} \rightarrow 0$, since there exists a minimum electric field below which a flashover is not possible.

JCM relation to the statistical model, we include in Table I values of $t_{89 \%}, A$, and the JCM constant $\gamma_{\mathrm{JCM}}$ for each flashover measurement. As described in Appendix A, $t_{89 \%}$ is the full width of the applied voltage pulse at $89 \%$ of its peak value, and $A$ is the actual (not projected) area of the vacuum-insulator interface. (Please see the last paragraph of Appendix A for a discussion of this area term.)

For the 17 PMMA measurements listed in Table I for which $0.5 \mathrm{~ns} \leq t_{89 \%} \leq 12 \mu$ s and $0.5 \leq d \leq 4.32 \mathrm{~cm}$, we find that the average value of the JCM constant $\gamma_{\mathrm{JCM}}$ is approximately 259 , and the standard deviation is 68 (26\%). Hence according to the JCM relation, the flashover probability is approximately $50 \%$ when

$$
E_{p} t_{89 \%}^{1 / 6} A^{1 / 10}=\gamma_{\mathrm{JCM}}=259 \pm 68 \text { (PMMA). }
$$

[The value of $\gamma_{\mathrm{JCM}}$ given by Eq. (18) is $48 \%$ higher than that estimated in Refs. [6,29]. Such a large discrepancy has also been noted in Refs. [36,37].] The fractional standard deviation given in Eq. (18) is a factor of 4 higher than is obtained with the statistical model [Eq. (16)], which suggests that these 17 measurements are more consistent with Eq. (16). The 90-s data listed in Table I also appear to be more consistent with Eq. (16). 
In Fig. 6, we plot $E_{p}$ as a function of $t_{89 \%}^{-1 / 6} A^{-1 / 10}$, the JCM scaling, for conditions under which the flashover probability is approximately $50 \%$, for the same data used to generate Fig. 5. Figures 5 and 6 also suggest that the measurements are in better agreement with the statistical model [Eq. (16)] than the JCM relation [Eq. (18)].

Comparing Eqs. (16) and (18), we see that the statistical model has a weaker time dependence $\left(t_{\mathrm{eff}}^{1 / 10}\right.$ vs $\left.t_{89 \%}^{1 / 6}\right)$ than the JCM relation. Table I and Figs. 5 and 6 suggest that $t_{\mathrm{eff}}^{1 / 10}$ scaling is in better agreement with experiment. This observation confirms the conclusion reached by Anderson [11], who finds that $t_{89 \%}^{1 / 6}$ scaling is obeyed for none of the measurements described in [11] that were performed with a $45^{\circ}$ insulator between ideal (flat) electrodes. This conclusion is based on the data plotted in Fig. 7 of Ref. [11], which appear to be significantly more consistent with $t_{\text {eff }}^{1 / 10}$ scaling. In addition, Eq. (18) assumes $t_{89 \%}$ and $C$ have different exponents; hence the JCM relation implicitly assumes that flashovers do not occur randomly in both time and space.

Since $A \propto d$, the statistical model and the JCM relation also have different functional dependencies on $d$ $\left[\exp (-0.24 / d)\right.$ vs $\left.d^{1 / 10}\right]$. The measurements performed by Yamamoto and co-workers $[20,22,23]$ suggest $d^{1 / 10}$

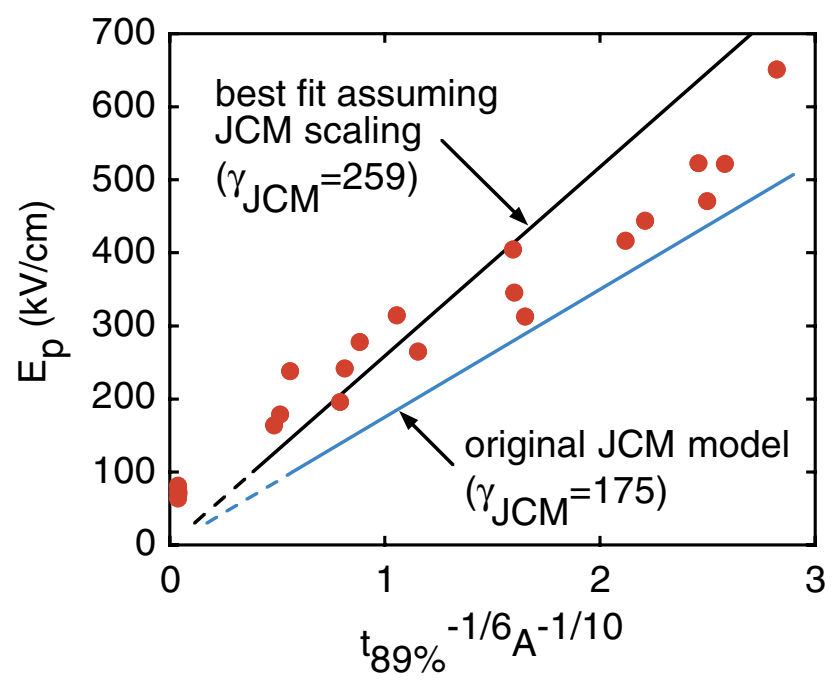

FIG. 6. (Color) The peak electric field $E_{p}$ as a function of $t_{89 \%}^{-1 / 6} A^{-1 / 10}$, which is the JCM scaling, for conditions under which the flashover probability is approximately 50\%. (The horizontal axis assumes $t_{89 \%}$ is expressed in $\mu \mathrm{s}$, and $A$ in $\mathrm{cm}^{2}$.) The figure plots the 17 PMMA measurements listed in Table I with $0.5 \mathrm{~ns} \leq t_{89 \%} \leq 12 \mu \mathrm{s}$ and $0.5 \leq d \leq 4.32 \mathrm{~cm}$, and the four measurements with $t_{89 \%}=90 \mathrm{~s}$. This is the same set of measurements used for Fig. 5. The fractional standard deviation in the values of $\gamma_{\mathrm{JCM}}$ for the 17 measurements is $26 \%$. The 90 -s measurements lie close together, and are the only measurements with $E_{p}<100 \mathrm{kV} / \mathrm{cm}$. The model becomes inapplicable as either $t_{89 \%} \rightarrow \infty$ or $A \rightarrow \infty$, which are equivalent to the limit $E_{p} \rightarrow 0$, since there exists a minimum electric field below which a flashover is not possible. scaling is not applicable for insulators with $0.5 \leq d \leq$ $1.5 \mathrm{~cm}$. As demonstrated in Table I, $\exp (-0.24 / d)$ scaling is in better agreement with these results. However, we note that $\exp (-0.24 / d)$ and $d^{1 / 10}$ scaling are, to within experimental uncertainties, equivalent over the range $1.5 \leq d \leq 6 \mathrm{~cm}$. Since $A \propto C$, both the statistical model and the JCM relation have the same dependence on $C$.

\section{The formative component of the flashover process}

The statistical flashover model developed in Secs. IIA and IIB assumes that the delay time $\tau_{\text {delay }}$ is dominated by its statistical component ( $\left.\tau_{\text {delay }} \sim \tau_{\text {stat }} \gg \tau_{\text {form }}\right)$. This work complements theoretical models of the formation of the flashover plasma, after it has been initiated. Such models are presented in $[3,9,13,15,46-66]$, references therein, and the review article by Anderson [24]. We include below a brief discussion of two of these: the Anderson-Brainard model for cathode-initiated flashover $[24,52,53]$, and the Anderson model [13,24] for anodeinitiated flashover. The literature suggests that both mechanisms can be relevant to a $45^{\circ}$ insulator, although it appears that an anode-initiated process dominates.

The Anderson-Brainard model for cathode-initiated flashover [24,52,53] applies when the flashover is initiated by electrons emitted from the cathode near the cathode triple junction. The model makes the following three assumptions: (i) Electrons strike the insulator surface repeatedly in a hopping motion [secondaryelectron-emission (SEE) coefficient of unity], rather than skimming the insulator surface within a desorbed gas layer, as assumed by Avdienko and Malev [49,50]. (ii) The electron-stimulated-desorption (ESD) coefficient is a few percent, rather than a factor of $\sim 170$ as obtained in $[49,50]$ from an interpretation of gas-desorption data reported by Avdienko and Kiselev [81]. (iii) The space charge of electrons that are electrostatically confined near the insulator surface (the SEE avalanche) reaches a maximum value that is self-consistent with a saturated surface charge on the insulator. [The ESD coefficient of a few percent discussed in assumption (ii) above is obtained in Refs. [52,53] from a reinterpretation of the Avdienko and Kiselev [81] data, according to assumption (i).]

The Anderson-Brainard model has no empirical parameters, and predicts that $E_{p} \propto \tau_{\text {form }}^{-1 / 2}$. Experiments performed with nominally $0^{\circ}$ insulators that have a slightly negative cone angle, designed to produce a well-defined source of electrons to minimize the statistical component of the delay time, confirm this proportionality and also indicate a fair quantitative agreement with the model's predictions [52,53]. The model has been successfully extended to $45^{\circ}$ insulators subject to a train of bipolar electric-field pulses, as described in [15]. Under these conditions, the periods of reversed polarity (equivalent to a $-45^{\circ}$ geometry) allow electrons emitted from the cathode during these periods to strike the insulator sur- 
face directly. This unusual source of electrons may have eliminated the large statistical time delay that otherwise would have been expected at the lower electric fields discussed in [15].

For an insulator with a large positive cone angle (such as $45^{\circ}$ ) subject to a unipolar pulse, it appears that an anode-initiated-flashover process dominates $[1,10,13,24]$. Under such conditions, a surface dendrite is formed that originates from a point on the insulator near the anode triple junction, then branches toward the cathode. For a $45^{\circ}$ insulator-electrode system, the electric field in the vacuum at the vacuum-insulator interface is higher at this junction than anywhere else along the interface; the field inside the insulator is also higher here than anywhere else inside the dielectric.

The Anderson model for anode-initiated flashover $[13,24]$ assumes that such a flashover begins when localized bulk-breakdown events at the vacuum-insulator interface near the anode triple junction eject plasma into the vacuum. (We expect that the bulk breakdown strength of an insulator is reduced near the vacuum-insulator interface, due to imperfections in the interface.) The bulk breakdowns may be precipitated by field emission of electrons from the insulator near the anode junction [13]. When the plasma comes into contact with the anode and is raised to the anode potential (or when the plasma emits electrons until it is raised to near the anode potential), the electric field is enhanced at the edge of the expanding plasma on the insulator surface. New generations of localized bulk breakdowns occur where the field exceeds the bulk breakdown strength of the insulator surface, and the discharge branches across the interface. The flashover is complete when branch tips reach the cathode. This model needs further development to estimate how the formative time of such a flashover process depends on the electric field.

\section{FLASHOVER OF AN INSULATOR STACK}

\section{A. The total-stack-flashover probability $F(t)$}

\section{Formal expression, assuming that transit-time and nonuniform-grading effects can be neglected}

The theoretical expression developed in Sec. IIA for the flashover probability of a single insulator ring [Eq. (7)] can be generalized to estimate the total-flashover probability of an insulator stack, i.e., the probability that all of the rings in an insulator stack flash. As in Sec. IIA, the discussion below assumes $\tau_{\text {stat }} \gg \tau_{\text {form }}$. We also assume that when an insulator ring flashes, ultraviolet radiation from the flashover plasma is prevented from initiating flashovers in any of the other stack rings.

We first calculate the total-flashover probability for a stack consisting of two insulators, then generalize the expression obtained to an arbitrary number.
We label the insulator rings in a two-ring system as 1 and 2. In this section (and Sec. IIIA2) we neglect nonuniform-grading effects; hence we assume that before either ring flashes, the total voltage applied to the stack $V(t)$ is divided evenly between the two rings. Consequently, the electric field $E(t)$ across each ring equals $V(t) / 2 d$, where $d$ is the thickness of each ring.

We consider first the case when ring 1 flashes before ring 2, and assume ring 1 flashes at time $t_{1}$. We neglect (in this section and Sec. IIIA2) transit-time effects, and assume that the instant ring 1 flashes, the total voltage is applied to the entire circumference of ring 2. This doubles the electric field across the unflashed ring.

According to Eq. (7), the probability $s_{1}\left(t_{1}\right)$ that ring 1 survives until time $t_{1}$ is given by

$$
s_{1}\left(t_{1}\right) \equiv 1-f_{1}\left(t_{1}\right) \equiv \exp \left\{\frac{-C}{k^{\beta}} \int_{0}^{t_{1}}\left[g_{1} E(\tau)\right]^{\beta} d \tau\right\},
$$

where $g_{1} \equiv 1$. (We include the $g_{1}$ factor here to facilitate generalizing the expression obtained to an arbitrary number of rings.) Assuming that $t_{2}>t_{1}$, the probability $s_{2}\left(t_{1}, t_{2}\right)$ that ring 2 survives until time $t_{2}$ is given by

$$
\begin{aligned}
& s_{2}\left(t_{1}, t_{2}\right) \equiv 1-f_{2}\left(t_{1}, t_{2}\right) \\
& \equiv \exp \left(\frac { - C } { k ^ { \beta } } \left\{\int_{0}^{t_{1}}\left[g_{1} E(\tau)\right]^{\beta} d \tau\right.\right. \\
&\left.\left.\quad+\int_{t_{1}}^{t_{2}}\left[g_{2} E(\tau)\right]^{\beta} d \tau\right\}\right) .
\end{aligned}
$$

The factor $g_{2} \equiv 2$, and accounts for the increase in the electric field across ring 2 after ring 1 flashes.

The probability that ring 1 survives until time $t_{1}$, then fails between $t_{1}$ and $t_{1}+\Delta t_{1}$, equals $\left[s_{1}\left(t_{1}\right)-s_{1}\left(t_{1}+\right.\right.$ $\left.\left.\Delta t_{1}\right)\right]$. Hence the probability that ring 1 survives until $t_{1}$ and fails between $t_{1}$ and $t_{1}+d t_{1}$ is obtained by differentiating Eq. (19) and equals $-\left[\partial s_{1}\left(t_{1}\right) / \partial t_{1}\right] d t_{1}=$ $\left[\partial f_{1}\left(t_{1}\right) / \partial t_{1}\right] d t_{1}$. Assuming that ring 1 fails at $t_{1}$, the probability ring 2 survives until time $t_{2}$, then fails between $t_{2}$ and $t_{2}+d t_{2}$, is obtained by differentiating Eq. (20) and equals $-\left[\partial s_{2}\left(t_{1}, t_{2}\right) / \partial t_{2}\right] d t_{2}=$ $\left[\partial f_{2}\left(t_{1}, t_{2}\right) / \partial t_{2}\right] d t_{2}$. Consequently, when ring 1 fails first, the probability that ring 1 survives until $t_{1}$ and fails between $t_{1}$ and $t_{1}+d t_{1}$, and ring 2 survives until $t_{2}$ and fails between $t_{2}$ and $t_{2}+d t_{2}$, is given by the following product:

$$
\frac{\partial f_{1}\left(t_{1}\right)}{\partial t_{1}} \frac{\partial f_{2}\left(t_{1}, t_{2}\right)}{\partial t_{2}} d t_{2} d t_{1}
$$

Integrating over all possible values of $t_{1}$ and $t_{2}$, we obtain the probability that both rings fail sometime between times 0 and $t$, assuming that ring 1 fails first:

$$
\int_{0}^{t} \frac{\partial f_{1}\left(t_{1}\right)}{\partial t_{1}}\left[\int_{t_{1}}^{t} \frac{\partial f_{2}\left(t_{1}, t_{2}\right)}{\partial t_{2}} d t_{2}\right] d t_{1}=\int_{0}^{t} \int_{t_{1}}^{t} \frac{\partial f_{1}}{\partial t_{1}} \frac{\partial f_{2}}{\partial t_{2}} d t_{2} d t_{1} .
$$

Since either ring can fail first, the probability $F(t)$ that 
both rings fail by time $t$ equals

$$
F(t)=2 \int_{0}^{t} \int_{t_{1}}^{t} \frac{\partial f_{1}}{\partial t_{1}} \frac{\partial f_{2}}{\partial t_{2}} d t_{2} d t_{1}
$$

Generalizing Eqs. (19)-(23) to an insulator stack consisting of $n$ rings, we obtain a formal expression for the probability that all of the rings in an $n$-insulator stack flash between times 0 and $t$ :

$$
F(t)=n ! \int_{0}^{t} \cdots \int_{\mathrm{t}_{n-2}}^{t} \int_{\mathrm{t}_{n-1}}^{t}\left(\prod_{i=1}^{n} \frac{\partial f_{i}}{\partial t_{i}}\right) d t_{n} d t_{n-1} \cdots d t_{1},
$$

where

$$
\begin{gathered}
f_{i}\left(t_{1}, t_{2}, \ldots t_{i}\right) \equiv 1-\exp \left(-\frac{C}{k^{\beta}} \sum_{j=1}^{i}\left\{\int_{t_{j-1}}^{t_{j}}\left[g_{j} E(\tau)\right]^{\beta} d \tau\right]\right), \\
g_{j} \equiv \frac{n}{n+1-j},
\end{gathered}
$$

and $t_{0} \equiv 0$.

\section{Analytic expression, assuming that transit-time and nonuniform-grading effects can be neglected}

For $n=1$, Eq. (24) reduces directly to Eq. (7). For $n=2$, we integrate Eq. (24) to obtain

$$
\begin{gathered}
F(t)=1-\frac{g_{2}^{\beta}}{g_{2}^{\beta}-2 g_{1}^{\beta}} \exp \left(-2 g_{1}^{\beta} R\right) \\
+\frac{2 g_{1}^{\beta}}{g_{2}^{\beta}-2 g_{1}^{\beta}} \exp \left(-g_{2}^{\beta} R\right),
\end{gathered}
$$

where

$$
R \equiv \frac{C}{k^{\beta}} \int_{0}^{t} E^{\beta}(\tau) \partial \tau=\frac{E_{p}^{\beta} t_{\mathrm{eff}} C}{k^{\beta}}=\frac{(\ln 2) E_{p}^{\beta} t_{\mathrm{eff}} C}{\left[\gamma_{\mathrm{SM}} \exp (\lambda / d)\right]^{\beta}} .
$$

When $n=3$, Eq. (24) can be expressed as follows:

$$
\begin{aligned}
F(t)= & -\frac{2 g_{2}^{\beta} g_{3}^{\beta}}{\left(2 g_{2}^{\beta}-3 g_{1}^{\beta}\right)\left(g_{3}^{\beta}-3 g_{1}^{\beta}\right)} \exp \left(-3 g_{1}^{\beta} R\right) \\
& +\frac{3 g_{1}^{\beta} g_{3}^{\beta}}{\left(2 g_{2}^{\beta}-3 g_{1}^{\beta}\right)\left(g_{3}^{\beta}-2 g_{2}^{\beta}\right)} \exp \left(-2 g_{2}^{\beta} R\right) \\
& -\frac{6 g_{1}^{\beta} g_{2}^{\beta}}{\left(g_{3}^{\beta}-3 g_{1}^{\beta}\right)\left(g_{3}^{\beta}-2 g_{2}^{\beta}\right)} \exp \left(-g_{3}^{\beta} R\right) .
\end{aligned}
$$

It is straightforward, but increasingly tedious, to integrate analytically Eq. (24) as $n$ increases. Equation (24) can be integrated numerically; however, when $n$ is large, this method requires a significant amount of time. For example, assuming $n=10$, that each integral is divided into 100 time steps, and that each time step for each integral can be completed in $10^{-9} \mathrm{~s}$, a straightforward numerical calculation of $F(t)$ [Eq. (24)] would require $\left(100^{10}\right)\left(10^{-9} \mathrm{~s}\right) \sim 3 \times 10^{3} \mathrm{y}$. Fortunately, we can use information about the form of the solution to develop an alternate approach that is more convenient when $n$ is large.

We first apply the alternate method to the case $n=2$. As indicated by Eq. (27), when $n=2$ the form of $F(t)$ is given by

$$
F(t)=1+a_{1} \exp \left(-2 g_{1}^{\beta} R\right)+a_{2} \exp \left(-g_{2}^{\beta} R\right)
$$

where $a_{1}$ and $a_{2}$ are constants. When $R \lll 1$, we can expand Eq. (30) as follows:

$$
\begin{aligned}
F(t)= & 1+a_{1}\left(1-2 g_{1}^{\beta} R+\frac{\left(2 g_{1}^{\beta} R\right)^{2}}{2}+\cdots\right) \\
& +a_{2}\left(1-g_{2}^{\beta} R+\frac{\left(g_{2}^{\beta} R\right)^{2}}{2}+\cdots\right) .
\end{aligned}
$$

When $R$ is sufficiently small, $F(t)$ must be proportional to $R^{2}$, since in this limit the flashover probability of each ring is proportional to $R$. Hence in this limit we obtain from Eq. (31) [and $F(t) \propto R^{2}$ ] the following two equations for $a_{1}$ and $a_{2}$ :

$$
\begin{gathered}
a_{1}+a_{2}=-1, \\
2 g_{1}^{\beta} a_{1}+g_{2}^{\beta} a_{2}=0 .
\end{gathered}
$$

Solving these equations produces a solution that is identical to Eq. (27). Of course, even though $R \lll 1$ was assumed to obtain the coefficients $a_{1}$ and $a_{2}$, the solution given by Eqs. (30), (32), and (33) is valid for arbitrary values of $R$, as is Eq. (27).

When $n=3$, this approach gives a solution that is identical to Eq. (29). For an arbitrary number of rings $n$, the form of $F(t)$ can be expressed as follows:

$$
F(t)=1+\sum_{j=1}^{n} a_{j} \exp \left[-(n+1-j) g_{j}^{\beta} R\right] .
$$

[This form of $F(t)$ is consistent with Eqs. (7), (27), and (29).] Since in the limit $R \lll 1$ we have $F(t) \propto R^{n}$, we find that the $a_{j}$ can be obtained from the following:

$$
\begin{gathered}
\underline{\mathbf{G}}\left(\begin{array}{c}
a_{1} \\
a_{2} \\
\vdots \\
a_{n}
\end{array}\right)=\left(\begin{array}{c}
-1 \\
0 \\
\vdots \\
0
\end{array}\right), \\
G_{i j}=\left[(n+1-j) g_{j}^{\beta}\right]^{i-1},
\end{gathered}
$$

where $\mathbf{G}$ is a second-rank matrix, and the $g_{j}$ are as defined by Eq. (26). Although we have assumed $R \lll 1$ to obtain the coefficients $a_{j}$, Eqs. (34)-(36) are valid for arbitrary values of $R$. 
As discussed above, when $R \ll 1$, then $F(t) \propto R^{n} \propto$ $\left(E_{p} / k\right)^{\beta n}$; hence in this limit $F(t)$ is a strong function of the ratio $E_{p} / k$.

A FORTRAN program has been written to solve Eqs. (34)-(36) and can be downloaded as described in Ref. [82]. On a typical workstation, the program computes $F(t)$ when $n=10$ in much less than $1 \mathrm{~s}$.

\section{Transit-time effects on $F(t)$, assuming nonuniform- grading effects can be neglected}

The expressions developed for the total-stack-flashover probability $F(t)$ in Secs. IIIA1 and IIIA2 [Eqs. (24) and (34)] assume that when a ring flashes, the resulting increased electric field across the remaining unflashed rings appears instantaneously around the entire circumference of the stack. This assumption is valid when $t_{\text {eff }}$ is much greater than the characteristic azimuthal transit time. [Equations (24) and (34) also assume that the effects of axial and radial transit times can be neglected.]

As discussed in [30], for many cases of practical interest, $t_{\mathrm{eff}}$ is less than the transit time. Under this condition, the effective circumference of the stack, and the flashover probability $F(t)$, are reduced. Following ideas described by Smith [30], we estimate in Appendix B the effects of nonzero transit time on $F(t)$. We find that these effects can be included by replacing the $g_{j}$ in Eqs. (25), (34), and (36) with the following expressions:

$$
\begin{gathered}
h_{1}^{\beta}=g_{1}^{\beta} \\
h_{2}^{\beta}=\left(1-\frac{\chi}{2}\right) g_{1}^{\beta}+\frac{\chi}{2} g_{2}^{\beta}, \\
h_{j}^{\beta}=\left(1-\frac{2(j-2) \chi}{j}-\frac{\chi}{j}\right) g_{1}^{\beta}+\frac{2 \chi}{j} \sum_{i=2}^{j-1} g_{i}^{\beta}+\frac{\chi}{j} g_{j}^{\beta}, \\
\text { for } j \geq 3,
\end{gathered}
$$

where

$$
\chi \equiv \frac{\nu t_{\mathrm{eff}}\left(10^{-6}\right)}{C},
$$

and $\nu$ is the propagation velocity of an electromagnetic pulse in the azimuthal direction around the stack. [The factor of $10^{-6}$ is included in Eq. (40) since we assume $t_{\text {eff }}$ is expressed in $\mu \mathrm{s}$.]

As indicated by the first term on the right-hand side of Eq. (39), this expression is valid only when $\chi \leq$ $n /(2 n-3)$, where $n$ is the number of insulators in the stack, since the coefficient of $g_{1}^{\beta}$ must be non-negative. Equation (39) also requires that $n \geq 3$. [When $n=2$, only Eqs. (37) and (38) are applicable; in this case, Eq. (38) requires that $\chi \leq 2$. When $n=1$, there are no transit-time effects, as long as the voltage is applied symmetrically to the single insulator ring.] When $\chi \gg$ $n /(2 n-3)$, transit-time effects can be neglected.

\section{Nonuniform-grading effects on $F(t)$, assuming transit-time effects can be neglected}

Equations (24) and (34) for the total-stack-flashover probability $F(t)$ assume that the voltage across the unflashed rings of a stack is always uniformly graded; i.e., they assume (i) that before any of the insulator rings in a stack flash, the voltage is evenly divided among the rings, and (ii) that whenever a ring flashes, the voltage is evenly divided among the remaining unflashed rings.

If the voltage is not always uniformly graded, the flashover probability $F(t)$ is increased. Following ideas presented by Smith [30], we estimate the effects of uneven voltage division in Appendix C. We find that, for an $n$-insulator stack, nonuniform grading can be approximately accounted for by replacing the $g_{j}$ in Eqs. (25), (34), and (36) with the following expressions:

$$
\begin{gathered}
g_{j, \mathrm{eff}}=\left[\frac{(j-1) !(n-j) !}{n !} \sum g_{j}^{\beta}\right]^{1 / \beta} \text { for } 1 \leq j<n, \\
g_{n, \mathrm{eff}}=g_{n}=n .
\end{gathered}
$$

The sum in Eq. (41) is over all possible $g_{j}$ factors, as discussed in Appendix C.

\section{Effects of both transit time and nonuniform grading on $F(t)$}

To estimate the effects of both transit time and nonuniform grading on $F(t)$, the $g_{j}$ in Eqs. (37)-(39) are replaced by the $g_{j \text {,eff }}$ defined by Eqs. (41) and (42), and the resulting values of $h_{j}$ are substituted for the $g_{j}$ in Eqs. (25), (34), and (36). (These effects are included in the FORTRAN code that can be downloaded as described in Ref. [82].)

\section{B. The auxiliary flashover probabilities $S(t)$ and $F_{\geq 1}(t)$}

Section IIIA2 develops an analytic expression for $F(t)$, the probability of a total-stack flashover. In this section, we develop two other analytic expressions that can be useful in certain applications.

Combining Eqs. (7) and (28), we obtain the survival probability $s(t)$ of a single insulator ring:

$$
s(t)=\exp (-R)
$$

Hence, if the voltage across an $n$-insulator stack is divided evenly among the rings before any of the rings flash, the probability $S(t)$ that all of the rings survive the application of a voltage pulse is readily obtained:

$$
S(t)=[s(t)]^{n}=\exp (-n R) .
$$

Consequently, the probability that at least one ring in an 
$n$-insulator stack flashes $F_{\geq 1}(t)$ can be expressed as

$$
F_{\geq 1}(t)=1-S(t)=1-\exp (-n R)
$$

Of course, $F_{\geq 1}(t)$ and $F(t)$ must satisfy

$$
F_{\geq 1}(t) \geq F(t) .
$$

If the initial voltage across a stack is applied symmetrically, azimuthal transit times have no effect on either $S(t)$ or $F_{\geq 1}(t)$, since these expressions depend only on the survival probability of each ring before any of the rings flash.

Equations (44) and (45) assume that the voltage across the stack is uniformly graded before any of the rings flash. When this condition is not met, the probability $S(t)$ that all of the rings in an $n$-insulator stack survive the application of a pulsed voltage is given by a generalization of Eq. (44):

$$
S(t)=\prod_{i=1}^{n} \exp \left(-g_{1, i}^{\beta} R\right)=\exp \left(-n g_{1, \mathrm{eff}}^{\beta} R\right),
$$

where $g_{1, i} E(t)$ is defined to be the field on the $i$ th ring before any of the rings flash. The quantity $E(t) \equiv V(t) / n d$ is the nominal electric field across each ring in an $n$-insulator stack before any of the rings flash, and $g_{1, \text { eff }}^{\beta}$ is as defined by Eq. (47). [ $g_{1, \text { eff }}^{\beta}$ is also defined by Eqs. (41) and (C8).] The values of the $g_{1, i}$ are readily obtained from a 2D electrostatic calculation of the voltage division among the stack rings before any of the rings flash. The probability that at least one ring flashes is then given by

$$
\begin{aligned}
F_{\geq 1}(t) & =1-S(t)=1-\prod_{i=1}^{n} \exp \left(-g_{1, i}^{\beta} R\right) \\
& =1-\exp \left(-n g_{1, \mathrm{eff}}^{\beta} R\right) .
\end{aligned}
$$

This expression for $F_{\geq 1}(t)$ is considerably simpler than the formal and analytic expressions for $F(t)$ [Eqs. (24) and (34)], and in certain situations can provide a meaningful upper bound to $F(t)$.

As discussed at the beginning of Sec. IIIA1, the expressions for $F(t)$ developed in Secs. IIIA1 and IIIA2
[Eqs. (24) and (34)] assume that ultraviolet radiation from a flashover plasma is not allowed to trigger a flashover in any of the unflashed rings of the stack. In the opposite limit, i.e., when UV radiation from the first ring that flashes is allowed to trigger immediate flashovers in all of the remaining rings, the expression for $F_{\geq 1}(t)$ given in Eq. (48) would be the total-stack-flashover probability.

\section{RESULTS}

\section{A. Comparison of predictions with the performance of one of the $Z$ stacks}

In this section and Table II, we compare predictions of the statistical flashover model developed in Secs. II and III with insulator-flashover measurements performed on one of the $Z$-accelerator insulator stacks.

$Z$ has 36 pulsed-power modules that deliver electromagnetic power to four insulator stacks at the accelerator's vacuum interface $[31,32,34-36,40-44]$. The source impedance of each module is $4.32 \Omega$. Nine modules deliver power to each of the four stacks; hence the impedance of each set of nine parallel modules is $0.48 \Omega$. The four stacks are also in parallel, and as a system operate at peak currents of $20 \mathrm{MA}$, peak voltages of $3 \mathrm{MV}$, and peak powers of $50 \mathrm{TW}$. Each of the upper two stacks includes five insulator rings connected in series; each of the lower two includes six rings. The two uppermost stacks are essentially identical; the uppermost stack is shown in Fig. 2.

The $45^{\circ} 5.72-\mathrm{cm}$-thick, $1003-\mathrm{cm}$-circumference insulator rings shown in Fig. 2 are fabricated from crosslinked polystyrene (Rexolite). The $0.95-\mathrm{cm}$-thick grading rings are hard-anodized aluminum [31]. The thickness of the anodized coating is $26 \pm 7 \mu \mathrm{m}$. The stack electrodes and grading rings are shaped to reduce the field at cathode triple junctions $15 \%$ below the value that would be obtained with flat electrodes. All insulator and electrode surfaces are machined to a roughness with a root-meansquare value $\leq 1.6 \mu \mathrm{m}$.

The stack is operated at nominal pressures between $1 \times$ $10^{-6}$ and $2 \times 10^{-5}$ torr, and is opened to atmospheric

TABLE II. Comparison of total-stack-flashover predictions with measurements for the upper-level Z-accelerator insulator stack.

\begin{tabular}{|c|c|c|c|c|}
\hline $\begin{array}{l}E_{p} \\
(\mathrm{kV} / \mathrm{cm})\end{array}$ & $\begin{array}{c}\text { Predicted } \\
\text { total-flashover } \\
\text { probability for } \\
\text { the upper-level } \\
Z \text { stack }\end{array}$ & $\begin{array}{l}\text { Range of predicted } \\
\text { probabilities, } \\
\text { assuming a } \pm 5 \% \\
\text { uncertainty in both } \\
E_{p} \text { and } \gamma_{\mathrm{SM}}\end{array}$ & $\begin{array}{l}\text { Range of predicted } \\
\text { probabilities, } \\
\text { assuming a } \pm 10 \% \\
\text { uncertainty in both } \\
E_{p} \text { and } \gamma_{\mathrm{SM}}\end{array}$ & $\begin{array}{c}\text { Measured } \\
\text { total-flashover } \\
\text { probability }\end{array}$ \\
\hline 100 & $3.5 \times 10^{-6}$ & $9.0 \times 10^{-8}-9.9 \times 10^{-5}$ & $1.8 \times 10^{-9}-1.9 \times 10^{-3}$ & $<5.0 \times 10^{-4}$ \\
\hline 158 & 0.26 & $0.043-0.75$ & $0.0038-0.99$ & $<0.2$ \\
\hline
\end{tabular}
The stack consists of five $45^{\circ} 5.72-\mathrm{cm}$-thick, $1003-\mathrm{cm}$-circumference Rexolite insulator rings connected in series. The flashover probabilities account for transit-time and nonuniform-grading effects, and were calculated using the FORTRAN program that can be downloaded as described in [82]. The large ranges in the third and fourth columns are due to the strong dependence of the flashover probability on the ratio $E_{p} / \gamma_{\mathrm{SM}}$. 
pressure and cleaned (with Scotch-Brite, ethyl alcohol, and paper towels) after every accelerator shot. The bottom insulator ring is the most exposed to debris from the load, and is regularly cleaned with 400-grit sandpaper. None of the stack components are oiled, as they are in many other pulsed-power accelerators. Most of the ultraviolet radiation produced by a flashover plasma is shielded from the rest of the stack by the grading rings, the magnetically insulated transmission line (MITL) anode shown in Fig. 2, and a debris shield not shown in the figure.

The calculated total-stack-flashover probabilities listed in Table II assume $\gamma_{\mathrm{SM}}=248$, as indicated by Eq. (17). The calculations account for transittime and nonuniform-grading effects as described in Secs. IIIA3-IIIA5, and Appendices B and C.

To account for transit time, we use Eqs. (37)-(39). To estimate $\nu$, the azimuthal velocity of an electromagnetic wave in the stack, we use the capacitance and inductance per unit length of the transmission line defined by two adjacent grading rings [30]. According to 2D electrostatic calculations, the total capacitance between two grading rings is $5 \mathrm{nF}$; hence the capacitance per unit length is $4.9 \times 10^{-10} \mathrm{~F} / \mathrm{m}$. The inductance per unit length, including edge effects, is approximately $3.4 \times 10^{-7} \mathrm{H} / \mathrm{m}$ [83]. Hence we estimate that the characteristic azimuthal wave velocity $\nu$ is $\left[\left(4.9 \times 10^{-10}\right)\left(3.4 \times 10^{-7}\right)\right]^{-1 / 2}=0.26 c$, where $c$ is the speed of light. The azimuthal velocity $\nu$ is much less than $c$ because the capacitance is significantly affected by the water indicated in Fig. 2. At frequencies of interest, the relative dielectric constant of water is 80 , and the speed of an electromagnetic wave in water is $0.11 c$.

To account for nonuniform voltage grading, we use Eqs. (41) and (42). The values of the $g_{j, \text { eff }}$ in Eq. (41) are calculated as described in Appendix C, using the results from a series of $2 \mathrm{D}$ electrostatic calculations performed on the geometry shown in Fig. 2. To obtain $g_{1 \text {,eff }}$, a 2D calculation was performed to determine how the voltage is divided among the five rings of the stack, before any of the rings flash. To obtain $g_{2, \text { eff }}$, five $2 \mathrm{D}$ calculations were performed, each with a different ring shorted. To obtain $g_{3, \text { eff }}$, ten 2D calculations were performed, each with a different pair of rings shorted. To obtain $g_{4, \text { eff }}$, ten 2D calculations were performed, each with a different set of three rings shorted. Using this approach, we find that for the stack outlined in Fig. 2, $g_{1, \text { eff }}=1.009 g_{1}, g_{2, \text { eff }}=$ $1.075 g_{2}, g_{3, \text { eff }}=1.104 g_{3}$, and $g_{4, \text { eff }}=1.035 g_{4}$, where $g_{1}$, $g_{2}, g_{3}$, and $g_{4}$ are the ideal values given by Eq. (26). Of course, as indicated by Eq. (42), $g_{5 \text {,eff }}=g_{5}=5$.

For each of the two peak electric fields $E_{p}$ given in Table II, we present the nominal predicted total-flashover probability, the range of predicted probabilities assuming $\pm 5 \%$ uncertainties in both $E_{p}$ and $\gamma_{\mathrm{SM}}$, and the range assuming $\pm 10 \%$ uncertainties. The ranges are large, because the probability is a strong function of the ratio $\left(E_{p} / k\right) \propto\left(E_{p} / \gamma_{\mathrm{SM}}\right)$. [As discussed in Sec. IIIA2, in the limit $R \lll 1$, the total-flashover probability $F(t)$ for an $n$-insulator stack is proportional to $R^{n} \propto\left(E_{p} / \gamma_{\mathrm{SM}}\right)^{\beta n}$.]

The upper two $Z$ stacks, which are essentially identical, were operated at a peak electric field $E_{p}$ of $\sim 100 \mathrm{kV} / \mathrm{cm}$ on $\sim 1000 \mathrm{Z}$ shots. Six $D$-dot and three $B$-dot monitors were used to measure the stack voltage and current, respectively $[36,84]$. The monitors are located in the stack anode at nine azimuthally equidistant locations; one of the monitors is outlined in Fig. 2. For these shots, the characteristic value of $t_{\text {eff }}$ as defined by Eq. (8) was nominally $0.033 \mu \mathrm{s}$. There were no totalstack flashovers for either of the two stacks over the course of these experiments; hence the measured totalflashover probability at $100 \mathrm{kV} / \mathrm{cm}$ is less than 1 in 2000 for the stack design presented in Fig. 2. As indicated in Table II, this observation is consistent with the calculated flashover probabilities.

On five consecutive $Z$ shots (\#334-\#338), the stack outlined in Fig. 2 was operated without either the anode or cathode of the MITL that is normally connected, i.e., with no load, to permit observation of the stack performance at an increased electric field [36]. Since these shots were taken with no MITL electrodes or any other load hardware, the magnetic field at the stack $(\sim 1 \mathrm{mT}$, due to displacement current) was orders of magnitude less than that required for magnetic flashover inhibition $[85,86]$. Since there were no MITL electrodes, there were also no sources of charged particles or ultraviolet radiation, except for those inherent to the stack itself when operated at high voltage. The stack was disassembled, cleaned, and rebuilt just before these shots. New Rexolite insulator rings were installed during the reassembly. However, although the grading rings, stack anode, and stack cathode were cleaned, these components were not replaced, and had been used on the previous 333 shots. On shots \#334-\#338 the stack was operated at pressures between $6 \times 10^{-6}$ and $1.8 \times 10^{-5}$ torr.

The peak electric field $E_{p}$ achieved on these five shots (\#334-\#338) was approximately $158 \mathrm{kV} / \mathrm{cm}$, and there were no total-stack flashovers. [The characteristic value of $t_{\text {eff }}$ for these shots as defined by Eq. (8) was $0.027 \mu \mathrm{s}$.] As indicated in Table II, this result is consistent with the calculated probabilities, assuming 5\% uncertainties in both $E_{p}$ and $\gamma_{\mathrm{SM}}$. Hence, it appears that the predictions of the statistical-flashover model developed in Secs. II and III are consistent with the performance of the $Z$ stack, at both 100 and $158 \mathrm{kV} / \mathrm{cm}$.

\section{B. Improving the flashover performance of a stack}

The model developed in Secs. II and III suggests at least two methods for improving the flashover performance of an insulator stack. We consider, for example, the stack outlined in Fig. 2. If the grading rings shown in this figure were extended radially outward into the water to increase the interring capacitance, the azimuthal wave 
velocity $\nu$ would be reduced. As suggested above, the minimum value of $\nu$ for this stack is $0.11 c$. When $\nu=$ $0.11 c$, we find that the nominal flashover probabilities at 100 and $158 \mathrm{kV} / \mathrm{cm}$ would be $5.4 \times 10^{-7}$ and 0.17 , respectively, significantly less than the nominal values given in Table II, which assume $\nu=0.26 c$.

The model also suggests that for a fixed stack height, there exists a number of insulator rings that optimizes the peak voltage that can be applied to the stack. We consider again the stack outlined in Fig. 2. The total axial height of the five $5.72-\mathrm{cm}$-thick insulator rings and four $0.95-\mathrm{cm}$ thick grading rings is $32.40 \mathrm{~cm}$. We assume this height is fixed, that the number of grading rings is always one less than the number of insulators, and that the thickness of each grading ring is held constant at $0.95 \mathrm{~cm}$. We also assume $t_{\mathrm{eff}}=0.033 \mu \mathrm{s}, C=1003 \mathrm{~cm}$, and $\gamma_{\mathrm{SM}}=248$, and that the grading of the stack is as nonuniform as discussed in Sec. IVA. In addition, we take transit-time effects into account, and assume $\nu=0.26 c$.

Under these conditions, we find that when the totalstack-flashover probability $F=1.0 \times 10^{-4}$, the number of insulators $n$ that would maximize the peak voltage is 7 . For $n=4,5,6,7$, and 8, the peak voltages (at which $F=$ $1.0 \times 10^{-4}$ ) are $3.11,3.16,3.17,3.18$, and $3.16 \mathrm{MV}$, respectively. Since the total insulator height decreases as the number of rings increases, the corresponding peak electric fields for these five cases are 105.4, 110.6, 114.6, 119.0 , and $122.9 \mathrm{kV} / \mathrm{cm}$. We note that although the voltage is maximized at $n=7$, the required operating field is significantly higher at $n=7$ than at $n=5$, and the flashover probability for the $n=7$ design is more sensitive to uncertainties in $E_{p}$ and $\gamma_{\mathrm{SM}}$.

\section{DISCUSSION}

\section{A. Limits of applicability}

The flashover model presented in Secs. II and III appears to be consistent with most of the measurements listed in Tables I and II. The tables suggest that the model is reasonably accurate when $100 \leq E_{p} \leq 651 \mathrm{kV} / \mathrm{cm}$, $0.5 \mathrm{~ns} \leq t_{\text {eff }} \leq 10 \mu \mathrm{s}, \quad 0.5 \leq d \leq 5.72 \mathrm{~cm}, \quad 7.85 \leq C \leq$ $1003 \mathrm{~cm}$, and $1 \leq n \leq 5$. The measurements listed in these tables do not include all physically reasonable combinations of the variables $E_{p}, t_{\mathrm{eff}}, d, C$, and $n$ within these ranges, but only a small subset. Nevertheless, the model may be useful for systems with combinations of parameters (within these ranges) that have not yet been tested. The model might also provide reasonable flashoverprobability estimates for systems with parameters slightly outside these ranges. However, we note that the model becomes inapplicable as either $t_{\text {eff }} \rightarrow \infty$ or $C \rightarrow \infty$, which are equivalent to the limit $E_{p} \rightarrow 0$, since there exists a minimum electric field below which a flashover is not possible.

We recommend that the model be checked, and if necessary modified, as additional flashover measure- ments are performed. In particular, we suggest that the values of $\beta, \lambda$, and $\gamma_{\mathrm{SM}}$ be adjusted when the set of available $45^{\circ}$-insulator-flashover data increases significantly.

As discussed previously, the model developed in Secs. II and III applies only to a clean undamaged insulator-electrode system. It does not apply to systems that have a significant defect, i.e., a location contaminated with debris or compromised by an imperfection at which flashovers repeatedly take place, and which prevents a random spatial distribution. For example, if over the course of many accelerator shots, an insulator stack in a high-power accelerator experiences a total flashover during the accelerator's primary power pulse frequently enough to cause significant damage, flashovers may begin to occur preferentially at the damaged location. Such flashovers would begin to occur more frequently than predicted by Secs. II and III, since the assumptions implicit in these predictions would no longer be valid. Hence it may be necessary to design an insulator stack to have an extremely small probability of total flashover over the desired lifetime of the system.

The flashover model has an additional limitation that is inherent and is due to the statistical nature of the flashover process. As discussed in Sec. IIIA2 and indicated in Table II, when the total-flashover probability is small, the model's predictions are extremely sensitive to the ratio $E_{p} / \gamma_{\mathrm{SM}}$. The strong sensitivity suggests that the design of an insulator stack should account for uncertainties in both $E_{p}$ and $\gamma_{\mathrm{SM}}$.

\section{B. Applicability to other systems}

The statistical flashover model presented in Secs. II and III is based on the following three assumptions: (i) The initiation of a flashover is a stochastic process, (ii) the characteristic statistical component of the flashover delay time is much greater than the flashover formative time, and (iii) the average rate at which flashovers occur is a power-law function of the instantaneous value of the electric field $E(t)$.

The general nature of these assumptions suggests that the flashover model developed in Secs. II and III can be applied to any system subject to a pulsed stress, for which the statistical component of the time to failure is much greater than the failure formative time. A single such system can be modeled as described in Sec. II, and several systems connected in series as described in Sec. III. (Of course, different values for $\beta$ and $\gamma_{\mathrm{SM}}$, and a different expression for $k$, would likely be required.) The model can be used to estimate the failure probability as a function of the applied stress, the temporal duration of the stress, and the size of the system. The model also naturally leads to a self-consistent definition of an effective pulse width [Eq. (8)] that can be used to compare the 
effects of applied stresses with different normalized time histories.

\section{ACKNOWLEDGMENTS}

The authors are extremely grateful to R. Altes, W. Beezhold, R. Brockman, S. Chantrenne, R. Clark, P. Corcoran, D. Dalton, T. Dinwoodie, G. Donovan, S. Drennan, G. Feltz, J. Fockler, T. Gilliland, C. Guthrie, D. Guthrie D. Heath, K. Hodge, T. Hughes, C. Jeffs, M. Johnson, K. Jones, J. Kellogg, M. Kernaghan, H. Kishi, J. Lee, R. Leeper, M. K. Matzen, H. McGovern, C. Mendel, Jr., L. P. Mix, W. Moore, M. Pelock, D. Petmecky, B. Peyton, J. Potter, J. Poukey, J. Powell, K. Prestwich, P. Reynolds, D. Rice, G. E. Rochau, J. H. Seamen, D. Seidel, A. Seth, A. Sharpe, J. Slopek, I. Smith, P. Spence, S. Summers, D. Van De Valde, R. Wavrik, D. Welch, L. Wilson, F. Zutavern, the Z and Saturn Operations Groups, Ktech Corporation, TitanPulse Sciences Division, Mission Research Corporation, C-Lec Plastics, Votaw Precision Technologies, Team Specialty Products, and Prodyn Technologies for invaluable contributions. We are also deeply indebted to F. Zutavern, K. Prestwich, and T. Cutler for graciously reviewing this article. Sandia is a multiprogram laboratory operated by Sandia Corporation, a Lockheed Martin Company, for the U.S. Department of Energy's National Nuclear Security Administration under Contract No. DEAC04-94AL85000.

\section{APPENDIX A: THE JCM INSULATOR- FLASHOVER RELATION}

In this appendix, we describe the JCM insulator-flashover relation [6,29], and examine the experimental basis upon which the relation is based.

The JCM relation for a single $45^{\circ}$ PMMA insulator can be expressed as follows $[6,29]$ :

$$
E_{p} t_{89 \%}^{1 / 6} A^{1 / 10}=\gamma_{\mathrm{JCM}},
$$

where $E_{p}$ is the peak value in time of the spatially averaged electric field $E(t) \equiv V(t) / d, V(t)$ is the voltage applied across the insulator, $d$ is the insulator thickness as defined in Fig. 1, $t_{89 \%}$ is the full width of the applied voltage pulse at $89 \%$ of its peak value, $A$ is the product of $d$ and the insulator circumference at its midplane (which is the projected area of the vacuum-insulator interface), and $\gamma_{\mathrm{JCM}}$ is the JCM constant. (The definition of $A$ given here is that which was originally used. As discussed at the end of this appendix, $A$ is by convention presently defined to be the actual area of the interface.)

According to Refs. [6,29], a $45^{\circ}$ PMMA insulator can be expected to flash when $E_{p} t_{89 \%}^{1 / 6} A^{1 / 10}$ [the left-hand side of Eq. (A1)] exceeds $\gamma_{\mathrm{JCM}}$. Following standard practice, we express $E_{p}$ in $\mathrm{kV} / \mathrm{cm}, t_{89 \%}$ in $\mu \mathrm{s}$, and $A$ in $\mathrm{cm}^{2}$. In these units, the value of $\gamma_{\mathrm{JCM}}$ is estimated in $[6,29]$ to be 175 . As discussed in $[6,29]$, Eq. (A1) is most applicable when $10 \leq t_{89 \%} \leq 200 \mathrm{~ns}$.

Equation (A1) is based on the eight measurements listed in Table III, which also appears as Table I in Ref. [6] and Table 9-I in [29]. These measurements were performed by Smith [1], Watson [3], Glock and Linke [4,5], and Milton [7].

Table III lists two measurements by Smith (at 0.03 and $0.3 \mu$ s pulse widths) and three by Glock and Linke (at $0.003,0.03$, and $0.30 \mu$ s pulse widths). However, Glock and Linke performed measurements only at $0.003 \mu \mathrm{s}$ $[4,5]$. The 0.03 and $0.30 \mu$ s data reported in $[4,5]$ were simply quoted from Smith's work; hence these two measurements appear twice in Table III. (The flashover fields listed in Table III for these two sets of numbers differ because Martin increased Smith's fields by $10 \%$, for reasons discussed in [6,29].)

The two measurements by Smith listed in Table III were conducted at $\sim 10^{-3}$ torr, considerably higher than pressures presently used in high-power accelerators. Figure xvii in Ref. [1] suggests that the flashover field obtained by Smith at a $0.3 \mu$ s pulse width would have been higher had the pressure been lower. (The measure-

TABLE III. The $45^{\circ}$-insulator-flashover data (as presented in Refs. [6,29]) that were used to develop the JCM flashover relation. As discussed in Appendix A, only two of these measurements [7] were performed with an uncoated $45^{\circ}$ PMMA insulator between flat uncoated electrodes, at a pressure $\leq 10^{-4}$ torr.

\begin{tabular}{lccccl}
\hline \hline \multicolumn{1}{c}{ Reference } & $\begin{array}{c}\text { Insulator } \\
\text { material }\end{array}$ & $\begin{array}{c}A \\
\left(\mathrm{~cm}^{2}\right)\end{array}$ & $\begin{array}{c}d \\
(\mathrm{~cm})\end{array}$ & $\begin{array}{c}E_{p} \\
(\mathrm{kV} / \mathrm{cm})\end{array}$ & $\begin{array}{l}t_{89 \%} \\
(\mu \mathrm{s})\end{array}$ \\
\hline Smith [1] & PMMA & 40 & 2.5 & 220 & 0.03 \\
& & & & 128 & 0.3 \\
Watson [3] & Lexan & 10 & 0.95 & $\sim 200$ & 0.07 \\
Glock and Linke [4,5] & PMMA & 25 & 1.25 & 290 & 0.003 \\
& & & & 200 & 0.03 \\
Milton [7] & & & 114 & 0.30 \\
& PMMA & 14 & 1.27 & 304 & 0.02 \\
& & & & 196 & 0.6 \\
\hline \hline
\end{tabular}


ments by Watson, Glock and Linke, and Milton were performed at pressures $\leq 10^{-4}$ torr.) In addition, the PMMA measurements described in Ref. [1] were performed with oiled insulators, whereas the Watson and Milton data were taken with unoiled samples. Glock and Linke performed measurements with and without oil, and report that applying a thin layer of oil to an insulator does not significantly change the flashover strength [4,5]. However, if an oil droplet forms on an insulator due to excess oil, the flashover strength can be reduced. For example, a 3-4 $\mathrm{mm}$ diameter droplet near the cathode can reduce the flashover strength $\sim 20 \%[4,5]$.

The Glock and Linke experiments were performed with a stainless-steel-mesh anode to provide optical-diagnostic access to the insulator surface $[4,5]$. Since the electric field was enhanced at the mesh-wire edges, we expect that the flashover field obtained in [4,5] would have been higher had a flat anode been used.

Watson's measurement was not performed with PMMA, and was not obtained at $45^{\circ}$ [3]. This flashover experiment was conducted with Lexan at $15^{\circ}$, and the flashover field obtained was increased $40 \%$ by Martin $[6,29]$ to approximate what the field would have been at $45^{\circ}$.

Hence, of the eight measurements listed in Table III which were used to develop the JCM flashover relation, only two (the measurements reported by Milton [7]) were performed with an uncoated $45^{\circ}$ PMMA insulator between flat uncoated electrodes at a pressure $\leq 10^{-4}$ torr. Consequently, only two are relevant to the design of a pulsed-power accelerator such as $Z$.

In addition, the $t_{89 \%}^{1 / 6}$ scaling predicted by Eq. (A1) is inconsistent with more recent measurements. For example, Anderson [11] finds that $t_{89 \%}^{1 / 6}$ scaling is obeyed in none of the experiments described in [11] that were conducted with flat electrodes. These and the results presented in Secs. IIB and IIC, and Figs. 5 and 6, suggest that the pulse-width scaling is significantly weaker than predicted by Eq. (A1).

We also note that each of the insulator areas listed in Table III is the projected area (as defined at the beginning of this appendix) of the vacuum-insulator interface. (This is not stated in [6,29], but can be inferred from the original references $[1,3-5,7]$.) However, the JCM relation is currently used [30,35] with the actual area of the interface, which is larger than the projected area by a factor of $2^{1 / 2}$. Furthermore, it appears that some of the pulse widths listed in Table III are incorrect. For example, the $0.03 \mu$ s value given for one of Smith's measurements is actually $0.015 \mu \mathrm{s}$ [87].

\section{APPENDIX B: TRANSIT-TIME EFFECTS ON $F(t)$, ASSUMING NONUNIFORM-GRADING EFFECTS CAN BE NEGLECTED}

The expressions developed for the total-stack-flashover probability $F(t)$ in Secs. IIIA1 and IIIA2 [Eqs. (24) and (34)] assume that when a ring flashes, the resulting increased electric field across the remaining unflashed rings appears instantaneously around the entire circumference of the stack. This assumption is valid when $t_{\text {eff }}$ is much greater than the characteristic azimuthal transit time. Under these conditions, and when nonuniform voltagegrading effects can be neglected, the $g_{j}$ factors in Eqs. (25), (34), and (36) are given by Eq. (26).

As discussed in [30], for many cases of practical interest, $t_{\text {eff }}$ is less than the transit time. Under this condition, the effective circumference of the stack, and the flashover probability $F(t)$, are reduced. Following ideas described by Smith [30], we estimate in this appendix the effects of a nonzero transit time on $F(t)$.

We first consider a uniformly graded stack consisting of two rings, as described in Sec. IIIA1. As in Sec. IIIA1, we consider the case when ring 1 flashes first. Assuming that the first ring flashes at time $t_{1}$, and that transit-time effects can be neglected, the probability $s_{2}\left(t_{1}, t_{2}\right)$ that the second ring survives until time $t_{2}$ is given by Eq. (20). When the characteristic value of $t_{2}-t_{1}$ is less than the azimuthal transit time, $s_{2}\left(t_{1}, t_{2}\right)$ is more correctly expressed as follows:

$$
s_{2}\left(t_{1}, t_{2}\right)=\exp \left\{-\frac{C}{k^{\beta}} \int_{0}^{t_{1}}\left[g_{1} E(\tau)\right]^{\beta} d \tau-\frac{1}{k^{\beta}} \int_{t_{1}}^{t_{2}}\left[C-2\left(\tau-t_{1}\right) \nu\right]\left[g_{1} E(\tau)\right]^{\beta} d \tau-\frac{1}{k^{\beta}} \int_{t_{1}}^{t_{2}} 2\left(\tau-t_{1}\right) \nu\left[g_{2} E(\tau)\right]^{\beta} d \tau\right\}
$$

where $\nu$ is the velocity of an electromagnetic pulse that travels in the stack in the azimuthal direction.

As indicated by Eq. (B1), the length of ring 2 that is subject to the field $g_{2} E(\tau)$ is zero at time $t_{1}$ (i.e., the instant ring 1 flashes), and increases thereafter linearly with time. The length of ring 2 that is subject to the initial field $g_{1} E(\tau)$ equals $C$ until time $t_{1}$, then afterward decreases linearly with time.

Assuming that $E(\tau)$ is constant over the effective width of the pulse $t_{\mathrm{eff}}$, and that characteristic values of $t_{1}$ and $t_{2}$ in the last two integrals of Eq. (B1) are $t_{\text {eff }} / 2$ and $t_{\text {eff }}$, respectively, we find that Eq. (B1) can be expressed as

$$
\begin{aligned}
s_{2}\left(t_{1}, t_{2}\right)=\exp \left(\frac { - C } { k ^ { \beta } } \left\{\int_{0}^{t_{1}}\left[h_{1} E(\tau)\right]^{\beta} d \tau\right.\right. \\
\\
\left.\left.+\int_{t_{1}}^{t_{2}}\left[h_{2} E(\tau)\right]^{\beta} d \tau\right\}\right),
\end{aligned}
$$

where 


$$
\begin{gathered}
h_{1}^{\beta}=g_{1}^{\beta}, \\
h_{2}^{\beta}=\left(1-\frac{\chi}{2}\right) g_{1}^{\beta}+\frac{\chi}{2} g_{2}^{\beta}, \\
\chi \equiv \frac{\nu t_{\mathrm{eff}}\left(10^{-6}\right)}{C} .
\end{gathered}
$$

The factor of $10^{-6}$ is included in Eq. (B5) since we express $t_{\text {eff }}$ in $\mu \mathrm{s}$.

For a stack with $n$ insulator rings, we use arguments similar to the above, and assume that all of the flashovers in the stack are aligned azimuthally, to obtain the following:

$$
\begin{gathered}
h_{1}^{\beta}=g_{1}^{\beta}, \\
h_{2}^{\beta}=\left(1-\frac{\chi}{2}\right) g_{1}^{\beta}+\frac{\chi}{2} g_{2}^{\beta}, \\
h_{j}^{\beta}=\left(1-\frac{2(j-2) \chi}{j}-\frac{\chi}{j}\right) g_{1}^{\beta}+\frac{2 \chi}{j} \sum_{i=2}^{j-1} g_{i}^{\beta}+\frac{\chi}{j} g_{j}^{\beta},
\end{gathered}
$$$$
\text { for } j \geq 3 \text {. }
$$

As indicated by the first term on the right-hand side of Eq. (B8), this expression is valid only when $\chi \leq$ $n /(2 n-3)$, where $n$ is the number of insulator rings, since the coefficient of $g_{1}^{\beta}$ must be non-negative. Equation (B8) also requires that $n \geq 3$. [When $n=2$, only Eqs. (B6) and (B7) are applicable; in this case, Eq. (B6) requires that $\chi \leq 2$. When $n=1$, there are no transit-time effects, as long as the voltage is applied symmetrically to the single insulator ring.] When $\chi \gg$ $n /(2 n-3)$, transit-time effects can be neglected.

To estimate the accuracy of Eqs. (B6) and (B7), we performed an exact calculation of $F(t)$ for a stack with $n=2$, using Eqs. (19), (23), and (B1). The calculation assumes $E(\tau)$ [Eqs. (19) and (B1)] is constant over the time interval $0 \leq \tau \leq t_{\text {eff }}$, and that $E(\tau)=0$ when $\tau<0$ and $\tau>t_{\text {eff }}$. We find that the exact failure probability is between 0 and $33 \%$ higher than the value obtained using Eqs. (B6) and (B7). [The difference is a function of the values of $F(t)$ and $\chi$.] An exact calculation performed for a stack with $n=3$ yields a failure probability that is between 0 and $20 \%$ higher than that obtained using Eqs. (B6)-(B8).

The effects of nonzero transit time can be included in the results of Secs. IIIA1 and IIIA2 by substituting the $h_{j}$ [Eqs. (B6)-(B8)] for the $g_{j}$ in Eqs. (25), (34), and (36).

\section{APPENDIX C: NONUNIFORM-GRADING EFFECTS ON $F(t)$, ASSUMING TRANSIT-TIME EFFECTS CAN BE NEGLECTED}

Equations (24) and (34) for the total-stack-flashover probability $F(t)$ assume that before any of the insulator rings in a stack flash, the voltage is divided evenly among the rings. The equations also assume that when one or more rings flash, the voltage is divided evenly among the remaining unflashed rings. Under these conditions, and when transit-time effects can be neglected, the $g_{j}$ factors in Eqs. (25), (34), and (36) are given by Eq. (26).

If the voltage is not always uniformly graded, the flashover probability $F(t)$ is increased. Following ideas presented by Smith [30], we estimate in this appendix the effects of uneven voltage division on $F(t)$.

We first consider a stack that consists of two rings, then generalize to a stack with an arbitrary number. The totalflashover probability for a uniformly graded two-ring stack is given by Eq. (27). This expression assumes that the probability ring 1 fails before ring 2 is the same as the probability ring 2 fails before ring 1 , which is valid when the voltage is evenly divided between the rings before either flashes.

If the voltage is not evenly divided, we must distinguish between the spatial position of each ring in the stack, and the temporal order in which each ring flashes. For the discussion in this appendix, we use letters to label the positions of the rings; hence we label the rings in a two-ring stack in spatial order as rings $a$ and $b$. We use numbers to label the temporal order in which a ring flashes; hence 2 refers to the second ring that flashes. Instead of using the $g_{j}$ factors given by Eq. (26), we define (for example) $g_{2(b, a)} E(t)$ to be the field on the second ring that flashes (during the time interval between when the first and second rings flash), when ring $b$ flashes first and $a$ flashes second. Using this notation, and following the arguments developed in Secs. IIIA1 and IIIA2, we find that the total-flashover probability for a two-ring stack can be expressed as follows:

$$
\begin{aligned}
F(t)= & \frac{1}{2}\left[1-\frac{g_{2(a, b)}^{\beta}}{g_{2(a, b)}^{\beta}-2 g_{1(a, b)}^{\beta}} \exp \left(-2 g_{1(a, b)}^{\beta} R\right)+\frac{2 g_{1(a, b)}^{\beta}}{g_{2(a, b)}^{\beta}-2 g_{1(a, b)}^{\beta}} \exp \left(-g_{2(a, b)}^{\beta} R\right)\right] \\
& +\frac{1}{2}\left[1-\frac{g_{2(b, a)}^{\beta}}{g_{2(b, a)}^{\beta}-2 g_{1(b, a)}^{\beta}} \exp \left(-2 g_{1(b, a)}^{\beta} R\right)+\frac{2 g_{1(b, a)}^{\beta}}{g_{2(b, a)}^{\beta}-2 g_{1(b, a)}^{\beta}} \exp \left(-g_{2(b, a)}^{\beta} R\right)\right] .
\end{aligned}
$$

The first term in square brackets is due to ring $a$ flashing before ring $b$, and the second is due to ring $b$ flashing before ring $a$.

Similar expressions can be developed for a stack with more than two rings, although the expressions become considerably more cumbersome as the number of rings is increased. We instead use an approximation, which we first 
discuss for the two-ring system. When there are only two rings, $g_{2(a, b)}=g_{2(b, a)}=2$, since after the first ring flashes, the entire voltage is applied across the remaining ring. However, since we assume that the stack is nonuniformly graded, $g_{1(a, b)}$ is not equal to $g_{1(b, a)}$.

We estimate $F(t)$ as given by Eq. (C1) for three cases. We first consider the situation when $2 g_{1(a, b)}^{\beta} R \ll 1$, $2 g_{1(b, a)}^{\beta} R \ll 1$, and $g_{2(a, b)}^{\beta} R=g_{2(b, a)}^{\beta} R \ll 1$. Under these conditions, we can approximate Eq. (C1) as follows:

$$
F(t)=g_{1, \mathrm{eff}}^{\beta} g_{2, \mathrm{eff}}^{\beta} R^{2},
$$

where

$$
\begin{gathered}
g_{1, \mathrm{eff}}^{\beta} \equiv \frac{g_{1(a, b)}^{\beta}+g_{2(b, a)}^{\beta},}{2} \\
g_{2, \mathrm{eff}}^{\beta}=g_{2(a, b)}^{\beta}=g_{2(b, a)}^{\beta}=2^{\beta} .
\end{gathered}
$$

When the grading is perfect, $2 g_{1}^{\beta} R \ll 1$, and $g_{2}^{\beta} R \ll 1$, we find from Eq. (27) that

$$
F(t)=g_{1}^{\beta} g_{2}^{\beta} R^{2},
$$

Since Eqs. (C2) and (C5) have the same form, they suggest that Eq. (C3) is a reasonable approximation for $g_{1, \mathrm{eff}}$, the effective value of $g_{1}$.

When $2 g_{1(a, b)}^{\beta} R \ll 1,2 g_{1(b, a)}^{\beta} R \ll 1$, and $g_{2(a, b)}^{\beta} R=$ $g_{2(b, a)}^{\beta} R \gg 1$, we can approximate Eq. (C1) as

$$
F(t)=1-\frac{g_{2, \text { eff }}^{\beta}}{g_{2, \text { eff }}^{\beta}-2 g_{1, \text { eff }}^{\beta}} \exp \left(-2 g_{1, \text { eff }}^{\beta} R\right) .
$$

[The coefficient of the exponent in Eq. (C6) is a good approximation, since $g_{2(a, b)}^{\beta}=g_{2(b, a)}^{\beta} \gg g_{1(a, b) \beta_{\beta}^{\beta}}^{\beta} g_{1(b, a)}^{\beta}$.] When the grading is perfect, $2 g_{1}^{\beta} R \stackrel{1}{\ll}$, and $g_{2}^{\beta} R \gg 1$, we find from Eq. (27) that

$$
F(t)=1-\frac{g_{2}^{\beta}}{g_{2}^{\beta}-2 g_{1}^{\beta}} \exp \left(-2 g_{1}^{\beta} R\right) .
$$

As Eqs. (C2) and (C5), Eqs. (C6) and (C7) suggest that Eq. (C3) is a reasonable approximation for $g_{1, \text { eff }}$.

When $2 g_{1(a, b)}^{\beta} R \gg 1,2 g_{1(b, a)}^{\beta} R \gg 1$, and $g_{2(a, b)}^{\beta} R=$ $g_{2(b, a)}^{\beta} R \gg 1$, then we obtain from Eq. (C1) that $F(t) \approx$ 1. This is the same result obtained from Eq. (27) when the grading is perfect, $2 g_{1}^{\beta} R \gg 1$, and $g_{2}^{\beta} R \gg 1$. Hence for the three cases considered, it appears that we can approximate $g_{1, \text { eff }}^{\beta}$ as indicated by Eq. (C3).

Using similar arguments for an $n$-insulator stack, we estimate that nonuniform voltage grading can be accounted for by replacing the $g_{j}$ in Eqs. (25), (34), and (36) with the following:

$$
g_{j, \mathrm{eff}}=\left[\frac{(j-1) !(n-j) !}{n !} \sum g_{j}^{\beta}\right]^{1 / \beta} \quad \text { for } 1 \leq j<n,
$$

$$
g_{n, \text { eff }}=g_{n}=n .
$$

The sum in Eq. (C8) is over all of the different possible $g_{j}$ factors for a stack; the number of the different possible $g_{j}$ factors is $n ! /(j-1) !(n-j) !$.

We illustrate the use of Eq. (C8) for a specific example. We consider a nonuniformly graded stack with three rings. We assume that before any of the rings flash, the electric field across ring $a$ is a factor of 1.05 higher than the nominal value of $V(t) / 3 d$, the field across ring $b$ is $0.97[V(t) / 3 d]$, and the field across ring $c$ is $0.98[V(t) / 3 d]$. We define $V(t)$ to be the voltage across the entire stack, and $d$ the thickness of each ring. This grading can be determined from a 2D electrostatic calculation of the voltage division among the rings of the stack, before any of the rings flash. Under these conditions we have

$$
g_{1, \mathrm{eff}}=\left\{\frac{\left[(1.05)^{\beta}+(0.97)^{\beta}+(0.98)^{\beta}\right]}{3}\right\}^{1 / \beta} .
$$

We also assume that if ring $a$ flashes first, the field across ring $b$ increases to a factor of 1.57 higher than the original nominal field $V(t) / 3 d$, and the field on $c$ increases to $1.43[V(t) / 3 d]$. If ring $b$ flashes first, we assume that the fields across rings $a$ and $c$ increase to factors of 1.40 and 1.60 higher than the original field, respectively. If ring $c$ flashes first, the factors for rings $a$ and $b$ are assumed to be 1.65 and 1.35 . These factors can be obtained from three $2 \mathrm{D}$ electrostatic calculations of the voltage division among the stack rings; each calculation would be performed with a different ring shorted. In this case we have

$$
g_{2, \text { eff }}=\left\{\frac{\left[(1.57)^{\beta}+(1.43)^{\beta}+(1.40)^{\beta}+(1.60)^{\beta}+(1.65)^{\beta}+(1.35)^{\beta}\right]}{6}\right\}^{1 / \beta} .
$$

Since the entire voltage is always applied to the last remaining unflashed ring, the $g$ factor for this ring is not affected by imperfect grading, and

$$
g_{3, \mathrm{eff}}=g_{3}=3 \text {, }
$$

as indicated by Eqs. (26), (42), and (C9).

Equations (C8)-(C12) are an oversimplification of considerably more complicated expressions, and provide use- ful approximations only when the $g_{j, \text { eff }}$ as defined by Eq. (C8) do not differ significantly from the ideal values $g_{j}$ given by Eq. (26).

[1] I. D. Smith, in Proceedings of the International Symposium on Insulation of High Voltages in Vacuum, 
edited by J. G. Trump, R. P. Little, E. M. Lyman, and A. S. Denholm (Massachusetts Institute of Technology, Cambridge, MA, 1964), p. 261.

[2] J. P. Shannon, S. F. Philp, and J. G. Trump, J. Vac. Sci. Technol. 2, 234 (1965).

[3] A. Watson, J. Appl. Phys. 38, 2019 (1967).

[4] W. R. Glock, Masters thesis, Cornell University, 1969.

[5] W. R. Glock and S. Linke, Cornell University Laboratory of Plasma Studies Report No. LPS 24, 1969.

[6] J.C. Martin, Atomic Weapons Research Establishment High Voltage Note No. 2, SSWA/JCM/713/157, 1971.

[7] O. Milton, IEEE Trans. Electr. Insul. 7, 9 (1972).

[8] O. Milton, Sandia National Laboratories Internal Memorandum, 25 April 1974.

[9] R. A. Anderson, in 1974 Annual Report of the Conference on Electrical Insulation and Dielectric Phenomena, Downington, PA, 1974, edited by the U.S. National Academy of Sciences (National Academy of Sciences, Washington, DC, 1975), p. 435.

[10] R. A. Anderson, in 1975 Annual Report of the Conference on Electrical Insulation and Dielectric Phenomena, Gaithersburg, MD, 1975, edited by the U.S. National Academy of Sciences (National Academy of Sciences, Washington, DC, 1978), p. 475.

[11] R. A. Anderson, Sandia National Laboratories Report No. SAND75-0667, 1976.

[12] R. A. Anderson, Proceedings of the VIIth International Symposium on Discharges and Electrical Insulation in Vacuum, Novosibirsk, USSR, 1976, edited by the USSR Academy of Sciences, Siberian Branch (USSR Academy of Sciences, Novosibirsk, USSR, 1976), p. 252.

[13] R. A. Anderson, in 1979 Annual Report of the Conference on Electrical Insulation and Dielectric Phenomena, Whitehaven, PA, 1979, edited by S. A. Boggs, C. M. Cooke, R. J. Densley, E. Sacher, and J. E. West (National Academy of Sciences, Washington, DC, 1979), p. 173.

[14] A. S. Pillai and R. Hackam, J. Appl. Phys. 56, 1374 (1984).

[15] R. A. Anderson and W. K. Tucker, J. Appl. Phys. 58, 3346 (1985).

[16] W. K. Tucker, R. A. Anderson, and D. E. Hasti, in Proceedings of the 5th IEEE Pulsed Power Conference, Arlington, VA, 1985, edited by P. J. Turchi and M. F. Rose (IEEE, Piscataway, NJ, 1985), p. 323.

[17] N.C. Jaitly and T.S. Sudarshan, in Proceedings of the 6th IEEE Pulsed Power Conference, Arlington, VA, 1986, edited by B. H. Bernstein and P. J. Turchi (IEEE, Piscataway, NJ, 1987), p. 64.

[18] N.C. Jaitly and T.S. Sudarshan, IEEE Trans. Electr. Insul. 22, 801 (1987).

[19] G. E. Vogtlin, W.W. Hofer, and M. J. Wilson, Lawrence Livermore National Laboratory UCRL Report No. 98704, 1988.

[20] O. Yamamoto, T. Hara, T. Nakae, and I. Ueno, in Proceedings of the XIIIth International Symposium on Discharges and Electrical Insulation in Vacuum, Paris, 1988, edited by J. M. Buzzi and A. Septier (Conservatoire National des Arts et Métiers, Paris, 1988), p. 250.
[21] G. E. Vogtlin and J. E. Vernazza, Proceedings of the 7th IEEE Pulsed Power Conference, Monterey, CA, 1989, edited by R. White and B.H. Bernstein (IEEE, Piscataway, NJ, 1989), p. 808.

[22] O. Yamamoto, T. Hara, T. Nakae, and M. Hayashi, IEEE Trans. Electr. Insul. 24, 991 (1989).

[23] O. Yamamoto, T. Hara, T. Nakae, I. Ueno, and M Hayashi (unpublished).

[24] R. A. Anderson, in Proceedings of the XIVth International Symposium on Discharges and Electrical Insulation in Vacuum, Santa Fe, NM, 1990, edited by R.W. Stinnett (Sandia National Laboratories, Albuquerque, NM, 1990), p. 311.

[25] G. E. Vogtlin, in Proceedings of the XIVth International Symposium on Discharges and Electrical Insulation in Vacuum, Santa Fe. NM, 1990 (Ref. [24]), p. 307.

[26] O. Yamamoto, T. Hara, I. Nakanishi, and M. Hayashi, in Proceedings of the XIVth International Symposium on Discharges and Electrical Insulation in Vacuum, Sante Fe, NM, 1990 (Ref. [24]), p. 400.

[27] O. Yamamoto, T. Hara, K. Ohmae, and M. Hayashi, Denki Gakkai Ronbunshi 110A, 830 (1990) [Electr. Eng. Jpn. 112, 11 (1992)].

[28] O. Yamamoto, T. Hara, H. Matsuura, M. Hayashi, and K. Yukimura, in Proceedings of the XVIth International Symposium on Discharges and Electrical Insulation in Vacuum, Moscow-St. Petersburg, 1994, edited by G. A. Mesyats (SPIE-International Society for Optical Engineering, Bellingham, WA, 1994), p. 354.

[29] J. C. Martin, in J. C. Martin on Pulsed Power, edited by T.H. Martin, A.H. Guenther, and M. Kristiansen (Plenum, New York, 1996), p. 255.

[30] I. D. Smith, IEEE Trans. Plasma Sci. 25, 293 (1997).

[31] H. C. Ives, D. M. Van De Valde, F.W. Long, J.W. Smith, R. B. Spielman, W. A. Stygar, R.W. Wavrick, and R.W. Shoup, in Proceedings of the 11th IEEE International Pulsed Power Conference, Baltimore, MD, 1997, edited by G. Cooperstein and I. Vitkovitsky (IEEE, Piscataway, NJ, 1997), p. 1602.

[32] M. A. Mostrom, T. P. Hughes, R. E. Clark, W. A. Stygar, and R. B. Spielman, in Proceedings of the 11th IEEE International Pulsed Power Conference, Baltimore, MD, 1997 (Ref. [31]), p. 460.

[33] I. S. Roth, P. S. Sincerny, L. Mandelcorn, M. Mendelsohn, D. Smith, T. G. Engel, L. Schlitt, and C. M. Cooke, in Proceedings of the 11th IEEE International Pulsed Power Conference, Baltimore, MD, 1997 (Ref. [31]), p. 537.

[34] R.W. Shoup, F. Long, T. H. Martin, R. B. Spielman, W. A. Stygar, M. A. Mostrom, K.W. Struve, H. Ives, P. Corcoran, and I. Smith, in Proceedings of the 11th IEEE International Pulsed Power Conference, Baltimore, MD, 1997 (Ref. [31]), p. 1608.

[35] I. D. Smith, P. A. Corcoran, W. A. Stygar, T. H. Martin, R. B. Spielman, and R.W. Shoup, in Proceedings of the 11th IEEE International Pulsed Power Conference, Baltimore, MD, 1997 (Ref. [31]), p. 168.

[36] W. A. Stygar, R. B. Spielman, R. A. Anderson, R. E. Clark, J.W. Douglas, T. L. Gilliland, M. L. Horry, T. P. Hughes, H. C. Ives, F.W. Long, T. H. Martin, D. H. McDaniel, O. Milton, M. A. Mostrom, J. F. Seamen, 
R.W. Shoup, J.W. Smith, K.W. Struve, G. E. Vogtlin, T. C. Wagoner, and O. Yamamoto, in Proceedings of the 12th IEEE International Pulsed Power Conference, Monterey, CA, 1999, edited by C. Stallings and H. Kirbie (IEEE, Piscataway, NJ, 1999), p. 454.

[37] J. M. Elizondo, A. J. Dragt, M. Krogh, B. Stygar, K. Struve, R. Spielman, and K. R. Prestwich, in Proceedings of the 13th IEEE International Pulsed Power Conference, Las Vegas, 2001, edited by R. Reinovsky and M. Newton (IEEE, Piscataway, NJ, 2001), p. 1810.

[38] O. Yamamoto, T. Takuma, Y. Kakehashi, S. Ikoma, A. Nishimoto, and T. Iida, in Proceedings of the XIXth International Symposium on Discharges and Electrical Insulation in Vacuum, Xi'an, China, 2000, edited by Xi'an Jiaotong University (Xi'an Jiaotong University, Xi'an, China, 2000), p. 108.

[39] W. A. Stygar, J. A. Lott, V. Anaya, H. C. Ives, T. C. Wagoner (to be published).

[40] R. B. Spielman, W. A. Stygar, J. F. Seamen, F. Long, H. Ives, R. Garcia, T. Wagoner, K.W. Struve, M. Mostrom, I. Smith, P. Spence, and P. Corcoran, in Proceedings of the 11th IEEE International Pulsed Power Conference, Baltimore, MD, 1997 (Ref. [31]), p. 709.

[41] P. A. Corcoran, J.W. Douglas, I. D. Smith, P.W. Spence, W. A. Stygar, K.W. Struve, T. H. Martin, R. B. Spielman, and H.C. Ives, in Proceedings of the 11th IEEE International Pulsed Power Conference, Baltimore, MD, 1997 (Ref. [31]), p. 466.

[42] R. J. Garcia, H. C. Ives, K. W. Struve, R. B. Spielman, T. H. Martin, M. L. Horry, R. Wavrik, and T. F. Jaramillo, in Proceedings of the 11th IEEE International Pulsed Power Conference, Baltimore, MD, 1997 (Ref. [31]), p. 1614.

[43] K.W. Struve, T. H. Martin, R. B. Spielman, W. A. Stygar, P. A. Corcoran, and J.W. Douglas, in Proceedings of the 11th IEEE International Pulsed Power Conference, Baltimore, MD, 1997 (Ref. [31]), p. 162.

[44] W. A. Stygar, R. B. Spielman, G. O. Allshouse, C. Deeney, D. R. Humphreys, H. C. Ives, F.W. Long, T. H. Martin, M. K. Matzen, D. H. McDaniel, C.W. Mendel, Jr., L. P. Mix, T. J. Nash, J.W. Poukey, J. J. Ramirez, T.W. L. Sanford, J. F. Seamen, D. B. Seidel, J.W. Smith, D. M. Van De Valde, R.W. Wavrik, P. A. Corcoran, J.W. Douglas, I. D. Smith, M. A. Mostrom, K. W. Struve, T. P. Hughes, R. E. Clark, R.W. Shoup, T. C. Wagoner, T. L. Gilliland, and B. Peyton, in Proceedings of the 11th IEEE International Pulsed Power Conference, Baltimore, MD, 1997 (Ref. 31]), p. 591.

[45] F. J. Zutavern, M.T. Buttram, and M.W. O'Malley, in Proceedings of the 16th Power Modulator Symposium, Arlington, VA, 1984, edited by K. Baile, S. Schneider, B. Gray, S. Levy, R. Pollard, and L. H. Klein (IEEE, Piscataway, NJ, 1984), p. 273.

[46] J. D. Cross and K. D. Srivastava, Appl. Phys. Lett. 21, 549 (1972).

[47] R. A. Anderson, Appl. Phys. Lett. 24, 54 (1974).

[48] R. A. Anderson, J. Appl. Phys. 48, 4210 (1977).

[49] A. A. Avdienko and M. D. Malev, Zh. Tekh. Fiz. 47, 1703 (1977) [Sov. Phys. Tech. Phys. 22, 986 (1977)].
[50] A. A. Avdienko and M. D. Malev, Vacuum 27, 643 (1977).

[51] K. D. Bergeron, J. Appl. Phys. 48, 3073 (1977).

[52] R. A. Anderson and J. P. Brainard, in Proceedings of the VIIIth International Symposium on Discharges and Electrical Insulation in Vacuum, Albuquerque, NM, 1978, edited by G.W. Kuswa, R. Bartsch, A. H. Guenther, J. A. Panitz, and M. Kristiansen (Sandia National Laboratories, Albuquerque, NM, 1978), p. D4-1.

[53] R. A. Anderson and J. P. Brainard, J. Appl. Phys. 51, 1414 (1980).

[54] J. E. Thompson, J. Lin, K. Mikkelson, and M. Kristiansen, Appl. Phys. Lett. 37, 574 (1980).

[55] A. S. Pillai and R. Hackam, J. Appl. Phys. 53, 2983 (1982).

[56] E.W. Gray, J. Appl. Phys. 58, 132 (1985).

[57] A. S. Pillai and R. Hackam, J. Appl. Phys. 58, 146 (1985).

[58] N. C. Jaitly and T. S. Sudarshan, J. Appl. Phys. 60, 3711 (1986).

[59] A. S. Pillai and R. Hackam, J. Appl. Phys. 61, 4992 (1987).

[60] N. C. Jaitly and T. S. Sudarshan, J. Appl. Phys. 64, 3411 (1988).

[61] R. G. Bommakanti and T. S. Sudarshan, J. Appl. Phys. 66, 2091 (1989).

[62] C. Le Gressus, F. Valin, M. Henriot, M. Gautier, J. P. Duraud, T.S. Sudarshan, R.G. Bommakanti, and G. Blaise, J. Appl. Phys. 69, 6325 (1991).

[63] G. Blaise and C. Le Gressus, J. Appl. Phys. 69, 6334 (1991).

[64] C. R. Li and T. S. Sudarshan, J. Appl. Phys. 76, 3313 (1994).

[65] A. Neuber, M. Butcher, L. L. Hatfield, and H. Krompholz, J. Appl. Phys. 85, 3084 (1999).

[66] O. Yamamoto, T. Takuma, M. Fukuda, S. Nagata, and T. Sonoda, IEEE Trans. Dielectr. Electr. Insul. 10, 550 (2003).

[67] R. Strigel, Elektrotech. Z 59, 33 (1938).

[68] J. M. Meek and J. D. Craggs, Electrical Breakdown of Gases (Clarendon, Oxford, 1953).

[69] J. D. Cobine, Gaseous Conductors (Dover, New York, 1958), pp. 186-193.

[70] F. Llewellyn-Jones, Ionization and Breakdown in Gases (Wiley, New York, 1966).

[71] Electrical Breakdown of Gases, edited by J. M. Meek and J. D. Craggs (Wiley, New York, 1978).

[72] Yu. D. Korolev and G. A. Mesyats, Physics of Pulsed Breakdown in Gases (URO, Yekaterinburg, 1998).

[73] R. C. Fletcher, Phys. Rev. 76, 1501 (1949).

[74] W. Weibull, J. Appl. Mech. 18, 293 (1951).

[75] T. H. Martin, IEEE Trans. Nucl. Sci. 18, No. 3, 104 (1971).

[76] O. Milton, Sandia National Laboratories Internal Memorandum, 5 March 1974.

[77] K. C. Kapur and L. R. Lamberson, Reliability in Engineering Design (Wiley, New York, 1977).

[78] Milton Abramowitz and Irene A. Stegun, Handbook of Mathematical Functions (Dover, New York, 1972).

[79] J. R. Taylor, An Introduction to Error Analysis (University Science, Sausalito, CA, 1997). 
[80] I. S. Roth et al. (unpublished).

[81] A. A. Avdienko and A.V. Kiselev, Zh. Tekh. Fiz. 37, 533 (1967) [Sov. Phys. Tech. Phys. 12, 381 (1967)].

[82] See EPAPS Document No. E-PRABFM-7-001407, which includes the FORTRAN source code that calculates the total-stack-flashover probability as estimated in Secs. II and III and Appendices B and C. A direct link to this document may be found in the online article's HTML reference section. The document may also be reached via the EPAPS homepage (http://www.aip.org/ pubservs/epaps.html) or from ftp.aip.org in the directory /epaps/. Please see the EPAPS homepage for more information.

[83] H. E. Knoepfel, Magnetic Fields (Wiley, New York, 2000), p. 369.
[84] W. A. Stygar, R. B. Spielman, H. C. Ives, W. B. S. Moore, J. F Seamen, A.W. Sharpe, T. C. Wagoner, T. L. Gilliland, R. S. Broyles, J. A. Mills, T. A. Dinwoodie, J. S. Slopek, K.W. Struve, and P. G. Reynolds, in Proceedings of the 11th IEEE International Pulsed Power Conference, Baltimore, MD, 1997 (Ref. [31]), p. 1258.

[85] J. P. VanDevender, D. H. McDaniel, E. L. Neau, R. E. Mattis, and K. D. Bergeron, J. Appl. Phys. 53, 4441 (1982).

[86] M. E. Savage, W. A. Stygar, J. M. Elizondo, H. C. Ives, K.W. Struve, and D. H. McDaniel, in Proceedings of the 2004 Power Modulator Conference, San Francisco, CA, 2004, edited by IEEE (to be published).

[87] I. D. Smith (unpublished). 